\title{
Monochromatic morphologies of Abell planetary nebulae ${ }^{\star}$
}

\author{
C.T. Hua ${ }^{1}$ and S. Kwok ${ }^{2}$ \\ 1 Laboratoire d'Astronomie Spatiale du CNRS, Allée Peiresc, Les Trois Lucs, BP. 8, 13376 Marseille Cedex 12, France \\ e-mail: trung.hua@astrsp-mrs.fr \\ 2 Department of Physics \& Astronomy, University of Calgary, Calgary, T2N 1N4, Canada \\ e-mail: kwok@iras.ucalgary.ca
}

Received February 26; accepted June 4, 1999

\begin{abstract}
Monochromatic $(\Delta \lambda \leq 10 \AA)$ images have been obtained for seven large $(2-10$ arcmin) Abell planetary nebulae. From this deep survey, we found 4 to have normal bipolar shapes, whereas 3 have truly spherical shells. The structures of these nebulae are discussed using the absolutely calibrated intensity maps in $\mathrm{H} \alpha,[\mathrm{N} \mathrm{II}]$, and [O III]. An attempt to derive ionized mass using our (dereddened) measured absolute flux as a function of distance to the $\mathrm{PN}$ is also given.
\end{abstract}

Key words: ISM: Planetary nebulae: individual (A 13, A 21, A 24, A 28, A 30, A 33, A 36)

\section{Introduction}

Planetary nebulae (PNe) are defined by their emission-line spectrum and often show extended nebulosities with definite morphological structures. However, $\mathrm{PNe}$ with large angular sizes are relatively rare. Among the $\approx 2000 \mathrm{PNe}$ catalogued (Perek \& Kohoutek 1967; Acker et al. 1992, etc.), less than $1 \%$ are larger than $10^{\prime}$. Large, extended $\mathrm{PNe}$ are often characterized by very faint brightness. They were first discovered and systematically studied by Abell (1966) using the Palomar Observatory Sky Survey plates. This pioneering work was later extended by Weinberger et al. (1983) who discovered more larger and fainter PNe. The extreme low surface brightness of these extended PNe has been attributed to the result of expansion. The Abell $\mathrm{PNe}$ are believed to be old and their central stars presumably have evolved past the constant-luminosity phase and are now on the cooling tracks. Because of their faintness, these PNe are likely to be discovered only if they are nearby, within the distances of $200-1000$ pc (Ishida \& Weinberger 1987).

Send offprint requests to: C.T. Hua

* The observations were carried out at the Observatoire de Haute Provence 120-cm telescope - CNRS, St Michel l'Observatoire, France.
Large surrounding haloes have also been detected in some of the Abell objects (e.g. A 65, A 66, Hua et al. 1998, and also via ftp://www.astrsp-mrs.fr/axesscientifiques/nebuleuses-planetaires). Recent development of large-format CCD detectors has made possible to imaging of these PNe with large field of view and high dynamical range. In this paper, we report new data consisting in narrow-band images of 7 Abell PNe and discuss possible relations between their morphologies and state of evolution.

\section{The observed sample}

$\mathrm{PNe}$ are dynamical systems existence of which requires the synchronization of the evolution between the central star and the nebula. During the lifetime of a PN, the central star is interacting both radiatively and dynamically with the nebula (Kwok 1994). An evolved (old) PN is usually defined by a high dynamical age of the nebula $\left(t_{\mathrm{dyn}}=R / V>10^{4} \mathrm{yr}\right.$, where $R$ and $V$ are respectively the radius and expansion velocity of the nebula). Since $t_{\mathrm{dyn}}$ is proportional to distance and reliable distances are often difficult to determine, old $\mathrm{PNe}$ can also be identified as objects with low surface brightness $\left(I=F / \pi \theta^{2}\right.$, where $F$ is the total flux from the nebula and $\theta$ is the angular radius). Since both $F$ and $\theta$ have the same dependence on distances $\left(\propto r^{-2}\right)$, the surface brightness criterion is therefore distance independent. Since circumstellar dust also disperses as the nebula evolves, evolved PNe often show less extinction and less infrared excess (Zhang \& Kwok 1993). These distance-independent parameters are also often used to distinguish young and old PNe. A quantitative measure of the infrared excess is the infrared excess index (IRE), defined as

$$
\begin{aligned}
\mathrm{IRE} & =\frac{L_{\mathrm{IR}}}{L\left(\mathrm{Ly}_{\alpha}\right)} \\
& =A \frac{F_{\mathrm{IR}}\left(10^{-11} \mathrm{erg} \mathrm{cm}^{-2} \mathrm{~s}^{-1}\right)}{F_{5 \mathrm{GHz}}(\mathrm{mJy})}
\end{aligned}
$$


where $F_{\mathrm{IR}}$ is the total amount of flux emitted in the infrared and the coefficient $A$ has the value of 1.0 and 1.5 at high and low densities respectively (e.g. Pottasch 1984; Zijlstra's thesis 1989). Young PNe usually have IRE values larger than 1, whereas old PNe have IRE values much less than 1. Although central stars of evolved $\mathrm{PNe}$ are expected to have high temperatures and low luminosity, these parameters are not necessarily related to the nebular age. Thus, a central star of high mass will evolve very fast $\left(t \propto M_{*}^{-10}\right)$, and will be on the cooling track even though the nebula is relatively young.

The PNe presented in this study are all selected from the Abell's (1966) catalogue. The only observations that have been reported were made with wide bandpass filters (Manchado et al. 1996). Since $\mathrm{H} \alpha$ and [N II] respond differently to nebular conditions, narrow-band observations separating these two emission lines will provide useful information on the nebular structure. The only object which was previously imaged through narrowband interference filters is A 13 (Rosado \& Moreno 1991), but these observations were made with 103 aG films coupled with an imagetube. In this paper, we report new observations through narrow-band filters and using a fast and high quality CCD detector. The coordinates and observed fluxes (when available in literature) are given in Table 1. Most of the line flux entries are taken from Cahn et al. (1992) and the Strasbourg-ESO Catalogue (Acker et al. 1992) as well. Strikingly, from this Table 1 and except for A 13, the observed $\mathrm{PNe}$ have the $\mathrm{H} \alpha / \mathrm{H} \beta$ intensity ratios rather smaller than the theoretical value of 2.87 in the recombination case B (e.g. Pottasch 1984), in contradiction with the optical extinction values quoted in Table 2 (Col. 5 , and taken from Cahn et al. 1992). For instance, considering A 28 and A 30 which apparently show no extinction $\left(c_{\alpha}=0.0\right)$, one should have obtained the predicted ratio (287) instead of 163 and 200 respectively. These cases need to be checked by further accurate spectrophotometric measurements. Furthermore, while most of the sample (except A 28 and A 30) have $5 \mathrm{GHz}$ radio fluxes measured, only two (A 30 and A 36) are bright enough in the far infrared to have IRAS measurements. In the case of A 30, most of the infrared emission comes from the inner part and not from the shell. If we except A 13 characterized by a rather strong extinction $\left(c_{\mathrm{H} \beta}(\right.$ radio $\left.)=1.47\right)$, all objects would satisfy the above criteria as being old PNe.

\section{The observations}

The observations were carried out with the CCD imager at the Observatoire de Haute Provence 120-cm telescope at St Michel l'Observatoire. The image scale and field coverage offered by the $\mathrm{f} / 6$ aperture and the Tektronix $1024 \times 1024$ CCD provide $0.686^{\prime \prime} / 24 \mu \mathrm{m}$-pixel and a large field of view of $11^{\prime} 7^{1}$. Images were bias subtracted and flat-fielded in the usual way. The flat fields were generally obtained in twighlight to ensure that the illumination across the detector was the same as the sky illumination in the observations. We have also used a $3 \times 3$ pixel window filtering to remove detector artifacts such as dead or noisy pixels, after correction for cosmic rays.

\subsection{The interference filters}

The interference filters used in the present study have very narrow bandwidths and are centred at specific wavelengths suitably selected (i.e. taking into account the $\mathrm{f} / 6$ aperture ratio of the $120-\mathrm{cm}$ telescope and the possible temperature variation during the nighttime) for investigating nebular ionization structure and/or the PN abundance distribution mainly $\mathrm{H} \alpha(\Delta \lambda=8.7 \AA)$, [N II $] 6583 \AA(\Delta \lambda=8.2 \AA)$, and [O III $] 5007 \AA(\Delta \lambda=$ $8.4 \AA)$. Narrow band filters are necessary to determine specific contribution from individual ion. As a matter of fact, some PNe do show [N II] $6583 \AA$ emission much stronger than H $\alpha$ 's ones (e.g. PN G 226.7+05.6 in Dopita \& Hua 1997). These filters are mounted directly in front of the detectors, in the direct imaging mode.

\subsection{The photometric calibration}

The CCD responses were calibrated by observing the standard stars HD 93512 and HD 109995. The O-type star was used for calibrating the red fluxes $(\mathrm{H} \alpha$ and [N II]6583 $\AA$ emission lines, to avoid the (weak) stellar absorption at the $\mathrm{H} \alpha$ line), while the blue flux calibration used the other standard star. Such a calibration was satisfactorily controlled by using the absolute flux calibration provided directly by compact PNe's measurements (Dopita \& Hua 1997; Hua et al. 1998). The image quality was typically $1^{\prime \prime} .5-22^{\prime \prime} 0$ during the observations. Nebular and standard star frames were processed and calibrated using either the IRAF or MIDAS software packages. The cleaned standard star frames then were used for the ADU counts/absolute flux conversion after airmass correction, sky subtraction and allowance for the filter bandwiths. Absolute fluxes (uncorrected for reddening) are given in erg $\mathrm{cm}^{-2} \mathrm{~s}^{-1}$ units (Table 2), with about $10 \%$ accuracy. However, further corrections for interstellar extinction might be eventually done using either optical or radio values quoted in Cols. $5 \& 6$ of the same table. In practice, once converted in absolute units, flux calculations were done in nebular frames with MIDAS INTEGRATE/APERTURE which allows to account for the actual contours of the planetary nebula (with $3 \sigma$ ) above the sky background. Such absolute flux

\footnotetext{
${ }^{1}$ Further details are available on the WEBsite http://www.obs-hp.fr
} 
Table 1. List of observed PNe

\begin{tabular}{|c|c|c|c|c|c|c|c|c|}
\hline Names & $\overline{\mathrm{PN} \mathrm{G}}$ & $\begin{array}{l}\alpha(2000) \\
(\mathrm{h} \mathrm{m} \mathrm{s})\end{array}$ & $\begin{array}{l}\delta(2000) \\
\left({ }^{\circ}, \prime \prime\right)\end{array}$ & $\mathrm{H} \alpha / \mathrm{H} \beta$ & $\begin{array}{l}\quad[\mathrm{NII}] / \mathrm{H} \beta \\
(\mathrm{H} \beta=100)\end{array}$ & {$[\mathrm{OIII}] / \mathrm{H} \beta$} & $\begin{array}{r}S(6 \mathrm{~cm}) \\
(\mathrm{mJy}) \\
\end{array}$ & $\begin{array}{r}-\log F\left(\mathrm{H}_{\beta}\right) \\
\left(\mathrm{erg} \mathrm{cm}^{-2} \mathrm{~s}^{-1}\right) \\
\end{array}$ \\
\hline A 13 & $204.02-08.52$ & 060446.3 & +035645 & $1580 \pm 950$ & $3980 \pm 1940$ (a) & $\ldots$ & $<26$ & 12.62 \\
\hline A 21 & $205.14+14.25$ & 072904.0 & +131446 & 250 & 425 & 147 & 327 & 10.40 \\
\hline A 24 & $217.18+14.76$ & 075138.5 & +030027 & 239 & 1518 & 105 & 55 & 11.35 \\
\hline A 28 & $158.81+37.19$ & 084136 & +581300 & 163 & 72 & $\ldots$ & $\ldots$ & 11.82 \\
\hline A 30 & $208.56+33.29$ & 084653.4 & +175246 & 200 & 1000 & 3000 & $\ldots$ & 12.19 \\
\hline A 33 & $238.03+34.87$ & 093910.5 & -024841 & 280 & $\ldots$ & 353 & 22 & 11.30 \\
\hline A 36 & $318.47+41.50$ & 134041.6 & -195242 & 100 & 32: & 117 & 215 & $10.86(b)$ \\
\hline
\end{tabular}

(a) The $\mathrm{H} \alpha$ and $\mathrm{H} \beta$ intensities are from Kaler (1983). (b) From Kaler et al. (1990). Values quoted in Acker et al. (1992). Note the surprisingly "small" values (except for A 13) of the Balmer decrement compared with the recombination case B prediction of 2.87 !

Table 2. Derived properties

\begin{tabular}{|c|c|c|c|c|c|c|c|c|c|}
\hline Object & $\log F(\mathrm{H} \alpha)$ & $\begin{array}{l}\log F([\mathrm{NII}]) \\
\left(\operatorname{erg} \mathrm{cm}^{-2} \mathrm{~s}^{-1}\right) \\
\end{array}$ & $\log F([\mathrm{OIII}])$ & $C_{\alpha}$ & $C_{\text {radio }}$ & $2 \theta_{\mathrm{H} \alpha}$ & $\begin{array}{c}2 \theta_{[\mathrm{NII}]} \\
(\operatorname{arcsec} \times \operatorname{arcsec})\end{array}$ & $2 \theta_{[\mathrm{OIII}]}$ & $\begin{array}{c}M_{i}(D / \mathrm{kpc})^{2.5} \\
\left(M_{\odot}\right)\end{array}$ \\
\hline A 13 & -11.215 & -11.117 & $\ldots$ & 2.2 & $1.47^{*}$ & $140 \times 205$ & $200 \times 220$ & weak & 0.57 \\
\hline A 21 & -10.466 & -10.398 & $\ldots$ & $\ldots$ & 0.35 & 550 & 550 & $\ldots$ & 2.42 \\
\hline A 24 & -10.870 & -10.396 & $\ldots$ & 0.48 & $0.53^{*}$ & 415 & $425 \times 550$ & $\ldots$ & 1.16 \\
\hline A 28 & $\ldots$ & $\ldots$ & $\ldots$ & 0.0 & $\ldots$ & 350 & 320 & not detected & $\ldots$ \\
\hline A 30 & -11.77 & -13.23 & -11.98 & 0.0 & $\ldots$ & 130 & knots & 130 & 0.05 \\
\hline A 33 & -11.43 & & -11.35 & 0.38 & -0.13 & 280 & not detected & 280 & 0.30 \\
\hline A 36 & -10.70 & $\ldots$ & $\ldots$ & 0.10 & 0.38 & $370 \times 490$ & not detected & $370 \times 490$ & 1.60 \\
\hline
\end{tabular}

For A 30, the [N II] flux is for the inner area including the 4 knots. Values quoted in Cols. 5 \& some of 6 are from Cahn et al. (1992) when available, otherwise we derived $c_{\mathrm{H} \beta}$ (radio) from $5 \mathrm{GHz}$ data and $\mathrm{H}_{\beta}$ flux from Acker92. Angular dimensions are measured in arc seconds on the $\mathrm{H}_{\alpha}$ images of observed PNe.

measurements are useful since $\mathrm{PN}$ fluxes are proportional to the emission measures $N_{\mathrm{e}}^{2} V$ (of course, we still miss the "depth" of the nebular volume, and the formula used assumes spheroidal structure!), it therefore provides quantitative density distributions of each of the observed ionic species.

\section{Morphology of the nebulae}

\subsection{A 13. $P N$ G 204.02-08.52}

Figure 2 shows the $\mathrm{H} \alpha$ image of A 13 . It shows an elliptical (or "skull-like" $105^{\prime \prime} \times 135^{\prime \prime}$ ) ring structure similar to that of NGC 6720 (Hua 1997; Guerrero et al. 1997). Our angular resolution $\left(28.65\right.$ arcsec $\mathrm{mm}^{-1}$ ) is much higher than that (49 $\operatorname{arcsec} \mathrm{mm}^{-1}$ ) of Rosado \& Moreno (1991), allowing us to better outline the inner "hole" of this new ring-nebula. The high ratio of the surface brightness between the ring and the "hole" suggests that this is not a projected spherical shell, but rather a bipolar nebula viewed pole-on, the line of sight being slightly off-axis, since the ionizing star is not exactly centred. The bright ring-structure is probably the high density equatorial waist of the bipolar nebula. As in NGC 6720, the nitrogen (inner) structure is slightly $\left(155^{\prime \prime} \times 114^{\prime \prime}\right)$ larger than the hydrogen distribution. The outer $[\mathrm{N}$ II] emission is definitely much more extended than that in $\mathrm{H} \alpha$, along with diffuse extensions over $\sim 12^{\prime}$ (Fig. 3 ), which displays a PA almost perpendicular to that of the bright ring. This result is quite in very good agreement with Rosado \& Moreno [N $\mathrm{NI}]$ image. Due to their use of a larger bandpass filter including $\mathrm{H} \alpha$ and [N II] lines, Manchado et al. (1996) were not able to outline these above features. Our observations instead enable us to discriminate specific contribution from each ion: e.g. Fig. 4 shows the $\left[\begin{array}{ll}\mathrm{N} & \mathrm{II}\end{array}\right] / \mathrm{H} \alpha$ intensity ratio obtained by superimposing the two monochromatic images. This ratio varies from 0.4 to 2.5 across the nebula (cross-section in Fig. 6), reflecting the changing ionization/excitation conditions of the nebula. Nitrogen is much more enhanced along the S-W part of the elliptical ring. $\left[\begin{array}{ll}\mathrm{O} & \mathrm{III}\end{array}\right]$ is rather faint (probably absent) since it is hardly detected against the sky background, even after 1 hour exposure. This result confirms the [O $\mathrm{O}$ III $]$ image of Manchado et al. The structure outlined in the intensity ratio map suggests that the apparent morphology of A 13 results from the projection on the plane of sky of a "wool-ball"-shape nebula, alike 
Sh 1-89 (see Hua et al. 1998), the bright elliptical waist being the equatorial area, with the fuzzy surrounding features.

\section{2. $A$ 21. $P N G 205.14+14.25$}

The filamentary appearance of A 21 has led to this object having been considered as a supernova remnant, $\mathrm{H}$ II region, Wolf-Rayet nebula, as well as PN (Arkhipova \& Lozinskaia 1978). A 21 has a PG 1159 central star and is classified by Napiwotzki \& Schönberner (1995) as spectral type $\mathrm{hgO}(\mathrm{C})$ in the scheme of Méndez et al. (1986) and DOZ in the white dwarf classification scheme of McCook \& Sion (1987). Since PG 1159 stars are believed to be transition objects between central stars of PNe and white dwarfs, there is little doubt that this object is an old PN. Spectroscopic observations of A 21 by Kwitter et al. (1983) show high $\mathrm{N} / \mathrm{O}$ and $\mathrm{He} / \mathrm{H}$ ratios, which is consistent of its being a Type I PN. The actual dimensions of this PN are much larger than our field of view $\left(11^{\prime} 7\right)$. According to Manchado et al. (1996, images given on p. 122), these could be even twice as large.

The $\mathrm{H} \alpha$ image, shown in Fig. 8, displays a network of filamentary structures in the S-E direction with fainter extensions completing a nearly spherical shell in the N-W. These filamentary structures are even more intense in the [N II] image (Fig. 9). The sizes of the nebula as measured from these two images are approximately the same, with a diameter of $\sim 550^{\prime \prime}$ The $[\mathrm{N} \mathrm{II}] / \mathrm{H} \alpha$ intensity ratio (Fig. 10) strongly varies $(0.6-1.2)$ from point to point with its highest value in the $\mathrm{N}-\mathrm{W}$ area. As a matter of fact, the two components are not distributed evenly throughout the nebula, reflecting differences in physical conditions, since the two emission lines depend upon different mechanisms. The complete ring (with the symmetry axis in the NW-SE direction) can clearly be seen, with a morphology bearing some resemblance to the double-ring structures seen in some PNe (e.g. MyCn 18, Sahai et al. 1998) as the result of the interacting winds process.

\section{3. $A$ 24. $P N$ G 217.18+14.76}

The $\mathrm{H} \alpha$ (Fig. 13) and [N II] images (Fig. 14) of A 24 show two strong elongated E-W lobes with a fragment of (W-side) arc which could be part of a helical structure. The $\mathrm{H} \alpha$ diffuse underlying emission has an irregular contour, along with an absorbing patch below the E lobe. In contrast, the $[\mathrm{N}$ II] image, noticeably larger, displays a double shell with remarkable radial structures outwards. These jet-like structures are similar to those observed in the $[\mathrm{N} \mathrm{II}]$ image of NGC 6543 (HST archives, program 5403, P. Harrington, P.I.). The patchy lane in the north shows up even more sharply. The two bright lobes could be the "waist" of a bipolar PN, with the diffuse underlying emission being the projection of two bubbles on top and below the waist. This structure is clearly evident in the $[\mathrm{N} \mathrm{II}] / \mathrm{H} \alpha$ intensity ratio image (Fig. 15). The jet-like radial structures, not apparent in wide bandpass images of Manchado et al. (1996, p. 125), are clearly seen in the NE and SW directions in (Fig. 14).

\subsection{A 28. $P N G 158.81+37.19$}

A 28 has a nearly circular shape of size $320^{\prime \prime}$ in the $\mathrm{H} \alpha$ image (Fig. 18) with the (rather faint) surface brightness distribution consistent of its being a sphere. In addition, a fragment of outer envelope (or shell) can be seen beyond the limb-brightened rim at the SW side extending to $\sim 360^{\prime \prime}$. The $[\mathrm{N}$ II] image (Fig. 19) is obviously smaller. However, nitrogen would suggest a more conspicuous bilobal structure (alike A 33 below but with much fainter brightness) than in the $\mathrm{H} \alpha$ image owing to a sort of void around the central star, which is not the case in $\mathrm{H} \alpha$. Figure 20 shows the [O III] image of A 28. [O III] is only weakly detected in this PN (undetected in Manchado et al. 1996, p. 109).

\subsection{A 30. $P N G$ 208.56+33.29}

A 30 consists of a normal outer envelope and a hydrogen deficient core (Jacoby \& Ford 1983). We have imaged A 30 in $\mathrm{H} \alpha,[\mathrm{N} \mathrm{II}]$, and [O III] and found the PN to have very different structures in the three images. The almost perfectly circular shell is most prominent in the $\mathrm{H} \alpha$ image (Fig. 22). We note the existence of four fragments of enhanced intensity along the outer edge of the shell. The same four enhancements along the rim can also be seen in the [O III] image (Fig. 24). The surface brightness distribution of the circular shell is consistent of it being a sphere. A series of "spider" features (first detected by Jacoby (1979) but shown in better details here) appear to whirl around the central knots. The inner core $\left(15^{\prime \prime} \times 18^{\prime \prime}\right.$ of A 30 has been imaged by the HST in [O III] and found to have spoke-like structures (Borkowski et al. 1995). The "spider" features lie outside of the spokes found in the HST image. The high velocity $\left(\sim 200 \mathrm{~km} \mathrm{~s}^{-1}\right)$ outflows detected by Meaburn \& López (1997 could arise from these "spider" features. Most interestingly, the shell is not detected in the $[\mathrm{N} \mathrm{II}]$ image, suggesting that the shell may be nitrogen poor. Figure 25 shows the inner regions of A 30 in $\mathrm{H} \alpha,[\mathrm{NII}]$ and [O III]. Two bright knots (in the NW and SE directions) close to the central star can be seen in all three images. Another knot (in the SW direction) is progressively stronger from $\mathrm{H} \alpha$, [N II] to [O III].

\subsection{A 33. $P N G 238.03+34.87$}

The signal-to-noise ratio of the present observations is much higher than in a previous report (Hua \& NguyênTrong 1983). The central star is classified as $\mathrm{O}(\mathrm{H})$ (low 
gravity and hydrogen rich) by Méndez (1992). By fitting the absorption line profiles by NLTE models, Méndez et al. (1985) derive an effective temperature of $100 \pm 3010^{3} \mathrm{~K}$ and a surface gravity of $\log g=6.0 \pm 0.5$. This is another example of perfectly spherical structure PN with the $\mathrm{H} \alpha$ (Fig. 27) and [O III] images (Fig. 28) showing similar structures. The intensity distribution is consistent with a 3 -D sphere, not a ring as in A 13. The inner region is rather non-uniform, giving an "owl"-shape. [N II] was not detected.

\subsection{A 36. $P N$ G $318.47+41.50$}

The present observations are of much better quality than in a previous report based on data obtained with a RCA image tube coupled with baked IIaO plates (Hua \& Nguyên-Trong 1983). A 36 has a bright $\left(m_{V}=11.5\right)$ central star and faint nebulosity. The central star is classified as $\mathrm{O}(\mathrm{H})$ by Méndez (1992). By fitting the absorption line profiles by NLTE models, Méndez et al. (1981) derive an effective temperature of $65 \pm 1010^{3} \mathrm{~K}$ and a surface gravity of $\log g=5.2 \pm 0.5$. Comparison with the Schönberner H-burning tracks yields a central star mass of $0.55 M_{\odot}$. The helical shape with a central elliptical ring is seen in both $\mathrm{H} \alpha$ and [O III] images, [N II] was not detected. Its morphology resembles that of NGC 6543 (Balick \& Preston 1987), showing two pairs of bipolar lobes. Features such as "rims" and "caps" can be seen in A 36 as in NGC 6543, using the terminology of Balick et al. (1994). The "caps" probably represent the region where the collimated outflows interact with the remnants of the circumstellar envelope of its asymptotic giant branch progenitor. The ROSAT X-ray spectrum of A 36 shows a two-component structure (Leahy et al. 1996). The high-energy (corresponding to a temperature of $710^{6} \mathrm{~K}$ ) component could be the result of this interaction. It would be interesting to take an image with an even larger field of view to see if the halo is present. Since A 36 is $\sim 15$ times larger than NGC 6543 in angular size, it must be much closer than NGC 6543 if the two objects are intrinsically similar. Given its galactic latitude of $41^{\circ}$, A 36 is probably no more than a couple hundred parsecs away.

\section{Physical properties}

The fact that these images are absolutely calibrated allows physical properties to be derived from these observations. The total nebular fluxes in $\mathrm{H} \alpha$, [N II], and [O III] are obtained by integrating over the nebular image for each of these filters. The corresponding logarithmic extinction values listed in Table 2 are taken from Cahn et al. 1992).

\subsection{Nebular masses}

The total flux $F_{0}(\mathrm{H} \alpha)$ emitted by the $\mathrm{H} \alpha$ line from a uniform density sphere of radius $R$ is given by

$F_{0}(\mathrm{H} \alpha)=\frac{4 \pi j_{\nu}}{4 \pi D^{2}}\left(\frac{4 \pi R^{3}}{3}\right)$

where $j_{\nu}$ is the line emission coefficient, and $D$ is the distance to the nebula. For a recombination line, $j_{\mathrm{H} \alpha}$ is often expressed in terms of the effective recombination coefficient

$\alpha^{\mathrm{eff}}=\frac{4 \pi j}{n_{\mathrm{e}} n_{\mathrm{p}} h \nu}$

where $n_{\mathrm{e}}$ and $n_{\mathrm{p}}$ are the electron and proton densities respectively. The flux in the recombination line can then be written as

$F_{0}(\mathrm{H} \alpha)=\left(\frac{R^{3}}{3 D^{2}}\right) h \nu_{\mathrm{H} \alpha} n_{\mathrm{e}} n_{\mathrm{p}} \alpha_{\mathrm{H} \alpha}^{\mathrm{eff}}$.

In practice, a filling factor $\epsilon$ is often used to take into account the actually observed nebular structures which are far from homogeneous (see morphologies displayed in a recent report by Hua et al. 1998). Its value lies between 0.3 and 0.7 . The ionized mass of the nebula is then given by

$M_{i}=\frac{4 \pi}{3} n_{\mathrm{p}} \mu m_{\mathrm{H}} \epsilon R^{3}$

where $\mu$ is the mean atomic mass per $\mathrm{H}$ atom. Combining Eqs. (5) and (6), the ionized nebular mass can be expressed in terms of the angular radius $(\theta)$ and $\mathrm{H}_{\alpha}$ flux as

$M_{i}=\frac{4 \pi \mu \mathrm{m}_{\mathrm{H}}}{\sqrt{3 h \nu_{\mathrm{H} \alpha} x_{\mathrm{e}} \alpha_{\mathrm{H} \alpha}^{\mathrm{eff}}}} \epsilon^{1 / 2} \theta^{3 / 2} D^{5 / 2} F_{0}(\mathrm{H} \alpha)^{1 / 2}$

where $x_{\mathrm{e}}=n_{\mathrm{e}} / n_{\mathrm{p}} \sim 1.16$. Since both $\theta$ and $F_{0}(\mathrm{H} \alpha)$ (i.e. our measured $F(\mathrm{H} \alpha)$ fluxes corrected for extinction using radio - or optical when radio data are missing $-c_{\mathrm{H} \beta}$ values quoted in Table 2) are measured in our images, the ionized masses for the nebulae can be obtained as a function of their distances. The effective recombination coefficients for the recombination lines of $\mathrm{H}$ are tabulated in Hummer \& Storey (1987). For example, $\alpha_{\mathrm{H} \alpha}^{\mathrm{eff}}$ at $T_{\mathrm{e}}=10^{4} \mathrm{~K}$ and $n_{\mathrm{e}}=10^{4} \mathrm{~cm}^{-3}$ has a value of $8.6510^{-14} \mathrm{~cm}^{3} \mathrm{~s}^{-1}$, and Eq. (7) can be written as

$M_{i}=0.032(\epsilon / 0.6)^{1 / 2} \theta^{3 / 2} D^{5 / 2} F_{0}(\mathrm{H} \alpha)^{1 / 2} M_{\odot}$

with $\theta$ in $\operatorname{arcmin}, D$ in $\mathrm{kpc}$, and $F_{0}(\mathrm{H} \alpha)=F(\mathrm{H} \alpha) \times$ $10^{0.693 \times c_{\mathrm{H} \beta}}$ in $10^{-12} \mathrm{erg} \mathrm{cm}^{-2} \mathrm{~s}^{-1}$ units. The derived values of $M_{i}$ (assuming $\epsilon=0.6$ ) are given in Table 2. We adopted the major axis values for elongated PNe (A 13, A 36). Given the uncertainties in the distances, the derived ionized masses (mainly less than $1 M_{\odot}$ ) for the other Abell $\mathrm{PNe}$ are not drastically different from most evolved PNe. 


\section{Discussion}

The Abell nebulae in this sample can be divided into two groups: truly spherical shells and bipolar nebulae viewed at different angles. Among the members of the first group are A 28, A 30, and A 33. A 13, although showing an elliptical shape, is not consistent with a spherical shell but rather represents a bipolar nebula viewed pole on. This planetary nebula would represent a "new ring" object owing to its great similarity with NGC 6720 . The remaining objects, A 21, A 24, and A 36 are bipolar nebulae similar to the general population of PNe. A systematic analysis of PNe images by Zhang \& Kwok (1998) has found that most PNe have similar intrinsic (bipolar) structures and their diverse appearances are mainly a function of sky orientation and ionization effects. This interpretation is supported by a recent deep CCD imaging where faint bipolar nebulosities are revealed in PNe (e.g. SaWe3, Hua et al. 1998) previously not classified as bipolar. In this context, the existence of perfectly spherical nebulae is puzzling. In the interacting winds shaping model of Balick (1987), PNe evolve from spherical to elliptical to butterfly shapes as the fast wind exploits and amplifies the slight asymmetries in AGB envelopes. In this model, the different morphologies of PNe represent different stages of the evolutionary sequence. However, the most spherical PNe, the best examples being A 28, A 30, and A 33 in the present sample, are often the oldest and most evolved PNe. It is quite possible that some other mechanism, other than interacting winds shaping, is at work. By comparing the morphologies of young and evolved PNe, Aaquist \& Kwok (1996) found that there is no significant difference in morphological class distribution between the two groups and suggested that the bipolar morphologies of PNe are created early in their evolution. From a sample of compact, young PNe observed by the HST, Sahai \& Trauger (1998) suggested that the bipolar morphologies of PNe are shaped by non-isotropic, collimated, fast ejections that develop in the proto-planetary nebula phase. The detection of jet-like features in A 24 is interesting, but it is uncertain whether these features are related to the collimated outflows that create the lobes that lie (presumably) perpendicular to the plane of the sky. The nature of A 30 is still controversial. It has been argued that the spherical shell of A 30 came from a primary isotropic ejection that occurred some $10^{4} \mathrm{yr}$ ago (the dynamical age derived from the shell expansion velocity) whereas the inner "ansae" were formed more recently $\left(\sim 10^{3}\right.$ yr $)$ by an ejection of hydrogen-depleted material (Jacoby \& Ford 1983). It has also been suggested that the second ejection is the result of "born-again" evolution where the PN has suffered a late thermal pulse and returned to the AGB (Iben et al. 1983). However, the fact that A 30 has undergone a He ignition episode does not explain why the primarily shell should be spherical. For some reason, the fast collimated flow must not have developed in A 30. Whether this is related to the mass of the central star is a question that remains to be answered.

\section{Conclusions}

New monochromatic images of 7 Abell PNe are presented. These images were absolutely calibrated, allowing the intensity distributions in different ions to be compared quantitatively. We concluded that 3 of the 7 Abell PNe are intrinsically spherical whereas the others have bipolar morphologies similar to the general PNe population. Haloes are detected outside of the shells of A 13 and A 24. We suggest that these haloes represent projections of the bipolar lobes on the plane of the sky. Jet-like, radial structures are also seen in A 24. The existence of a higher frequency of spherical morphologies among evolved $\mathrm{PNe}$ is still unexplained by current theories of PNe evolution.

Acknowledgements. CTH would like to thank the University of Calgary's staff for their kind hospitality greatly appreciated during his visit of the Department of Physics and Astronomy. Thanks are also expressed to Mme J. Martinis for her valuable assistance for using MIDAS/IDL facilities for image processing. The manuscript has been improved thanks to pertinent comments from Dr M. Rosado, the referee. This work is achieved for the GDR 968 "Milieux Circumstellaires".

\section{References}

Aaquist O.B., Kwok S., 1996, ApJ 462, 813

Abell G.O., 1966, ApJ 144, 259

Acker Ochsenbein F., Stenholm B., Tylenda R., Marcout J., Schön C., 1992, The Strasbourg-ESO Catalogue of Galactic Planetary Nebulae. ESO Publishing (Acker92)

Arkhipova V.P., Lozinskaia T.A., 1978, Sov. Astr. Lett. 4, 7

Balick B., 1987, AJ 94, 671

Balick B., Preston H.L., 1987, AJ 94, 958

Balick B., Perinotto M., Maccioni A., Terzian Y., Hajian A., 1994, ApJ 424, 800

Borkowski K.J., Harrington J.P., Tsvetanov Z.I., 1995, ApJ 449, L143

Cahn J.H., Kaler J.B., Stanghellini L., 1992, A\&AS 94, 399

Guerrero M.A., Manchado A., Chu Y.-H., 1997, ApJ 487, 328

Hua C.T., Nguyên-Trong T., 1983, A\&A 117, 272

Hua C.T., 1997, A\&AS 125, 355

Hua C.T., Dopita M.A., Martinis J., 1998, A\&AS 133, 361

Hummer D.G., Storey P.J., 1987, MNRAS 224, 801

Iben I., Kaler J.B., Truran J.W., Renzini A., 1983, ApJ 264, 605

Ishida K., Weinberger R., 1987, A\&A 178, 227

Jacoby G., 1979, PASP 91, 754

Jacoby G.H., Ford H.C., 1983, ApJ 266, 298

Kaler J.B., 1983, ApJ 271, 188

Kaler J.B., Shaw R.A., Kwitter K.B., 1990, ApJ 359, 392

Kwitter K.B., Jacoby G.H., Lawrie D.G., 1983, PASP 95, 732

Kwok S., 1994, PASP 106, 344

Leahy D.A., Zhang C.Y., Volk K., Kwok S., 1996, ApJ 466, 352 
Manchado A., Guerrero M., Stanghellini L., Serra-Ricart M., 1996, The IAC Morphological Catalog of Northern Galactic Planetary Nebulae

McCook G.P., Sion E.M., 1987, ApJSS 65, 603

Meaburn J., López J.A., 1997, ApJ 472, L45

Méndez R.H., 1992, in IAU Symp. 145 Evolution of Stars: The Photospheric Abundance Connection, Michaud G., Tutukov A. (eds.). Kluwer: Dordrecht, p. 375

Méndez R.H., Kudritzki R.P., Simon K.P., 1985, AAP 142, 289

Méndez R.H., Kudritzki R.P., Gruschinske J., Simon K.P., 1981, AAP 101, 323

Méndez R.H., Miguel C.H., Heber U., Kudrizki R.P., 1986, in IAU Coll. 87: Hydrogen deficient stars and related objects, Hunger K., Schönberner D., Kamesara Rao N. (eds.). Reidel: Dordrecht, p. 323

Napiwotzki R., Schönberner D., 1995, AAP 301, 545

Perek L., Kohoutek L., 1967, Catalogue of Galactic Planetary Nebulae, Prague, Academia, Prague

Rosado M., Moreno M.A., 1991, A\&AS 88, 245

Sahai R., et al., 1998, ApJ (submitted)

Sahai R., Trauger J.T., 1998, AJ 116, 1357

Weinberger R., Dengel J., Sabbadin F., 1983, ApJ 265, 249

Zhang C.Y., Kwok S., 1993, ApJSS 88, 137

Zhang C.Y., Kwok S., 1998, ApJSS 117, 341 


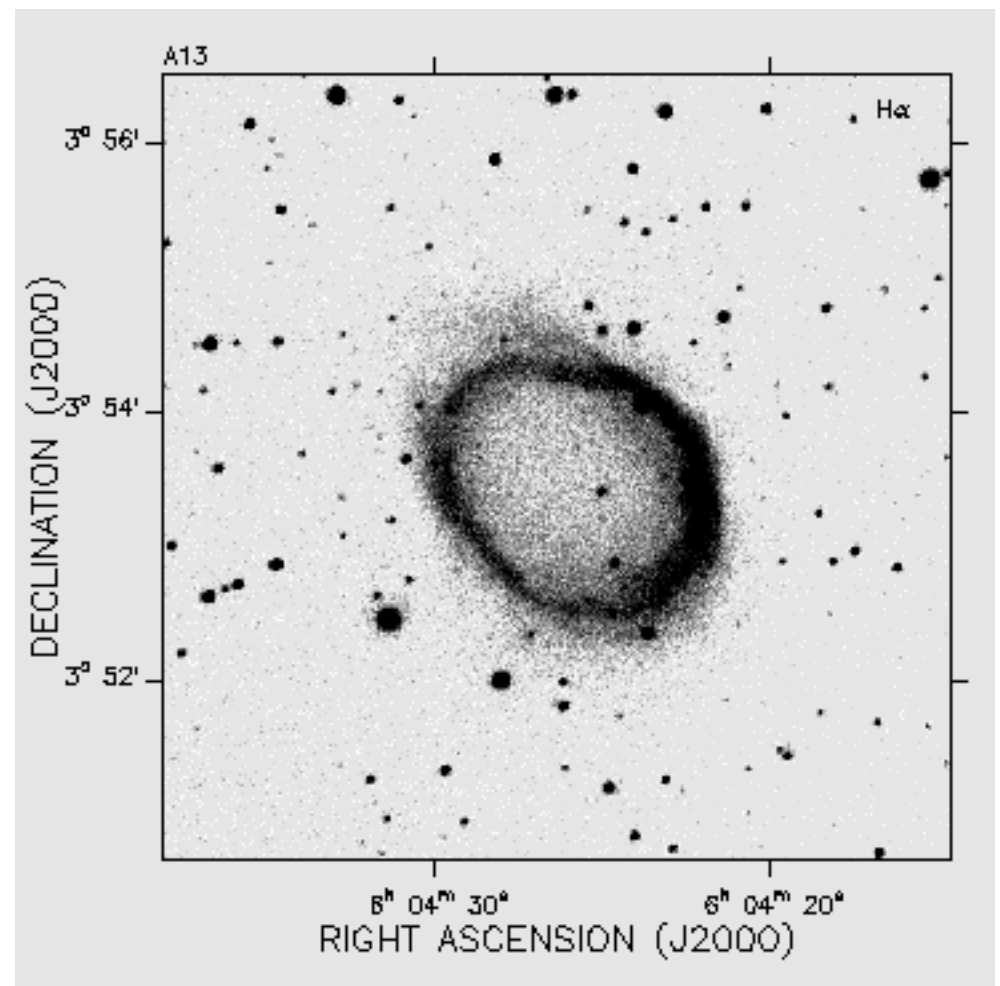

Fig. 1. A 13 in $\mathrm{H} \alpha$ with equatorial coordinates

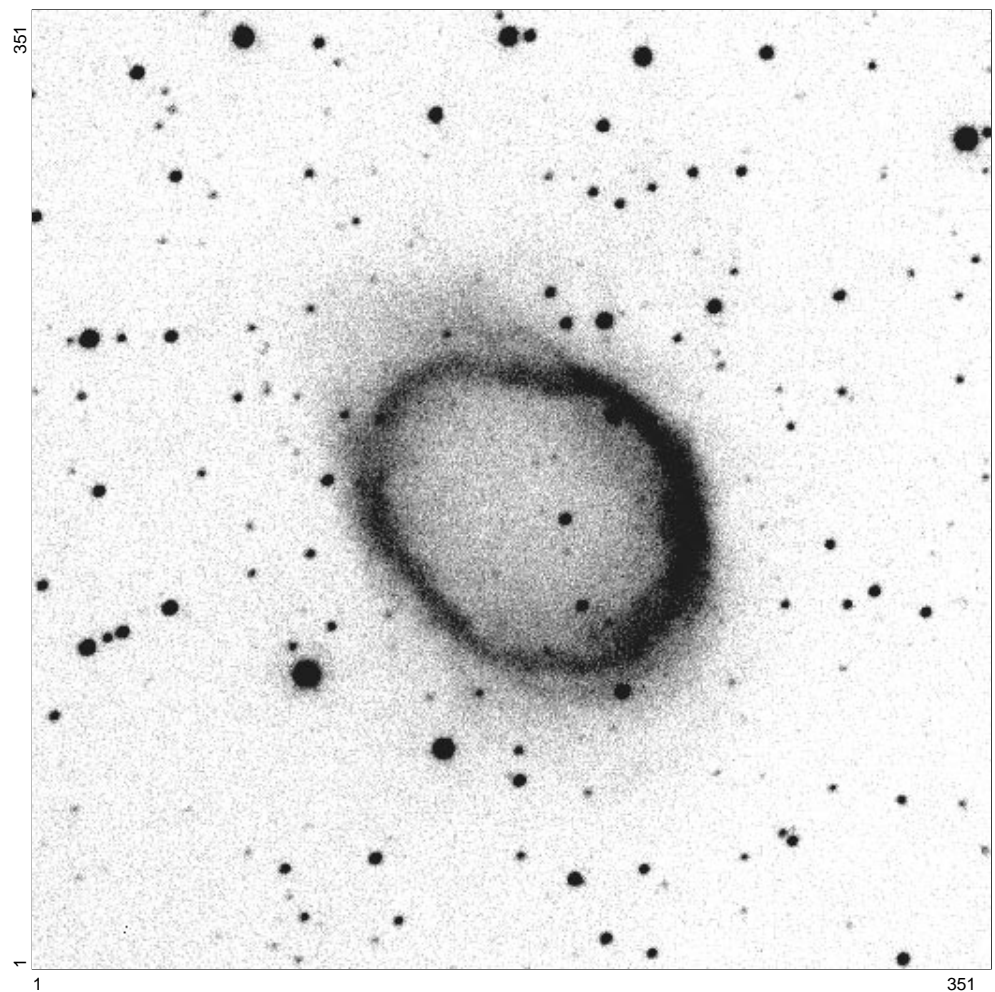

Fig. 2. This $\mathrm{H} \alpha$ image of A 13 displays an elliptical ring which resembles that of NGC 6720 . However, the outer emission is by far less extended. The image frame covers 350 square arc seconds. North is at the top, East to the left for all figures, unless otherwise stated 


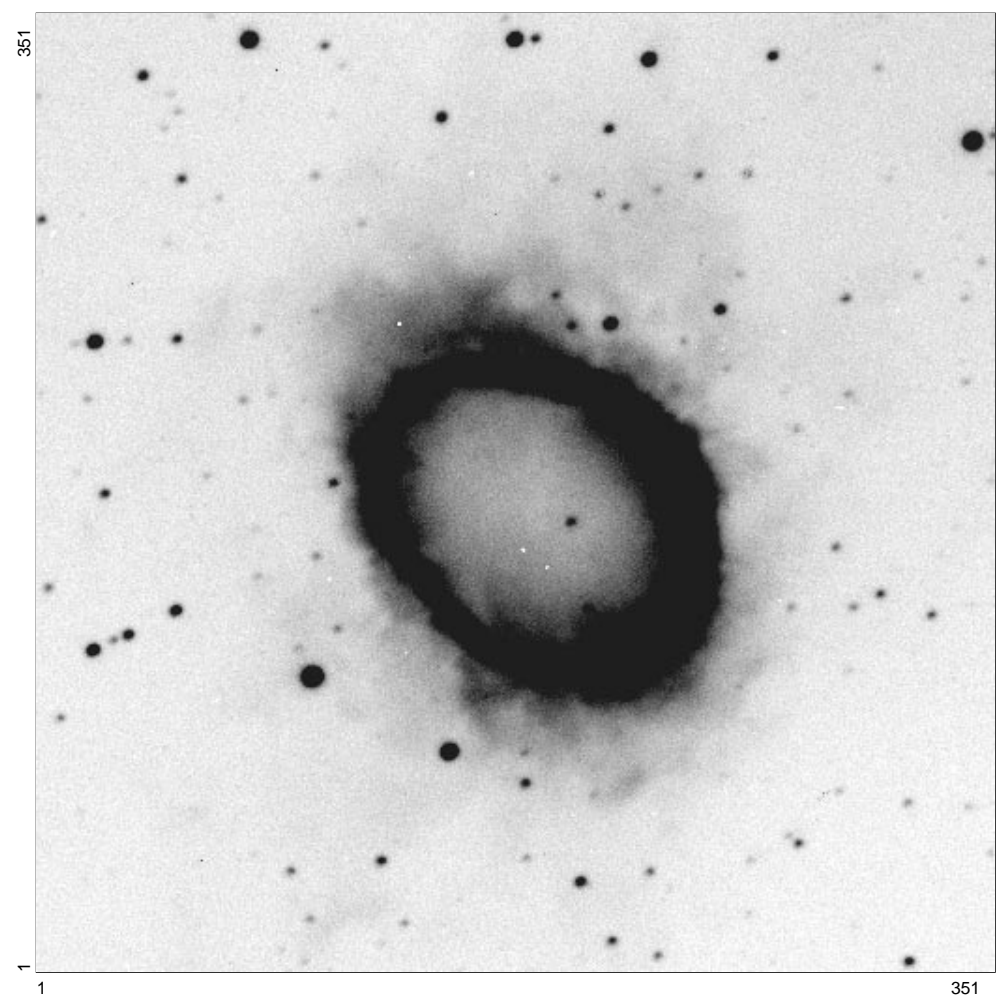

Fig. 3. The $\left[\mathrm{N}_{\mathrm{II}}\right]$ image of A 13, represented in the same image frame scale as for $\mathrm{H} \alpha$. Diffuse extensions can be seen spreading to radii as large as $\sim 12^{\prime}$, comparable to that given by Rosado \& Moreno (1991). The faint fuzzy outward radial structures could result from a projection of bubble-like emissions - like in Sh1-89 (Hua \& al. 1998) on the plane of sky. In nitrogen, A 13 appears as a multiple-shell planetary nebula

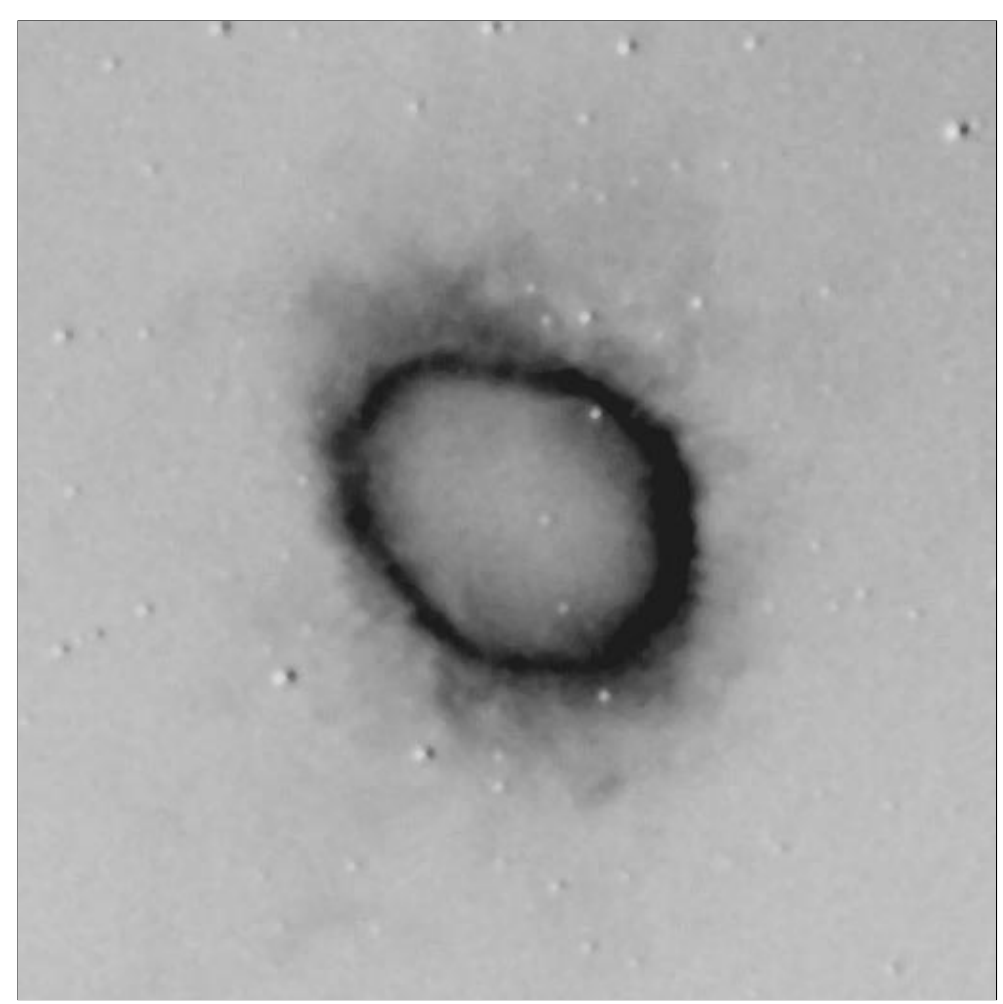

Fig. 4. Variation of the $[\mathrm{NII}]$ to $\mathrm{H} \alpha$ intensity ratio with a maximum of 2.5 in the $\mathrm{SW}$ area 


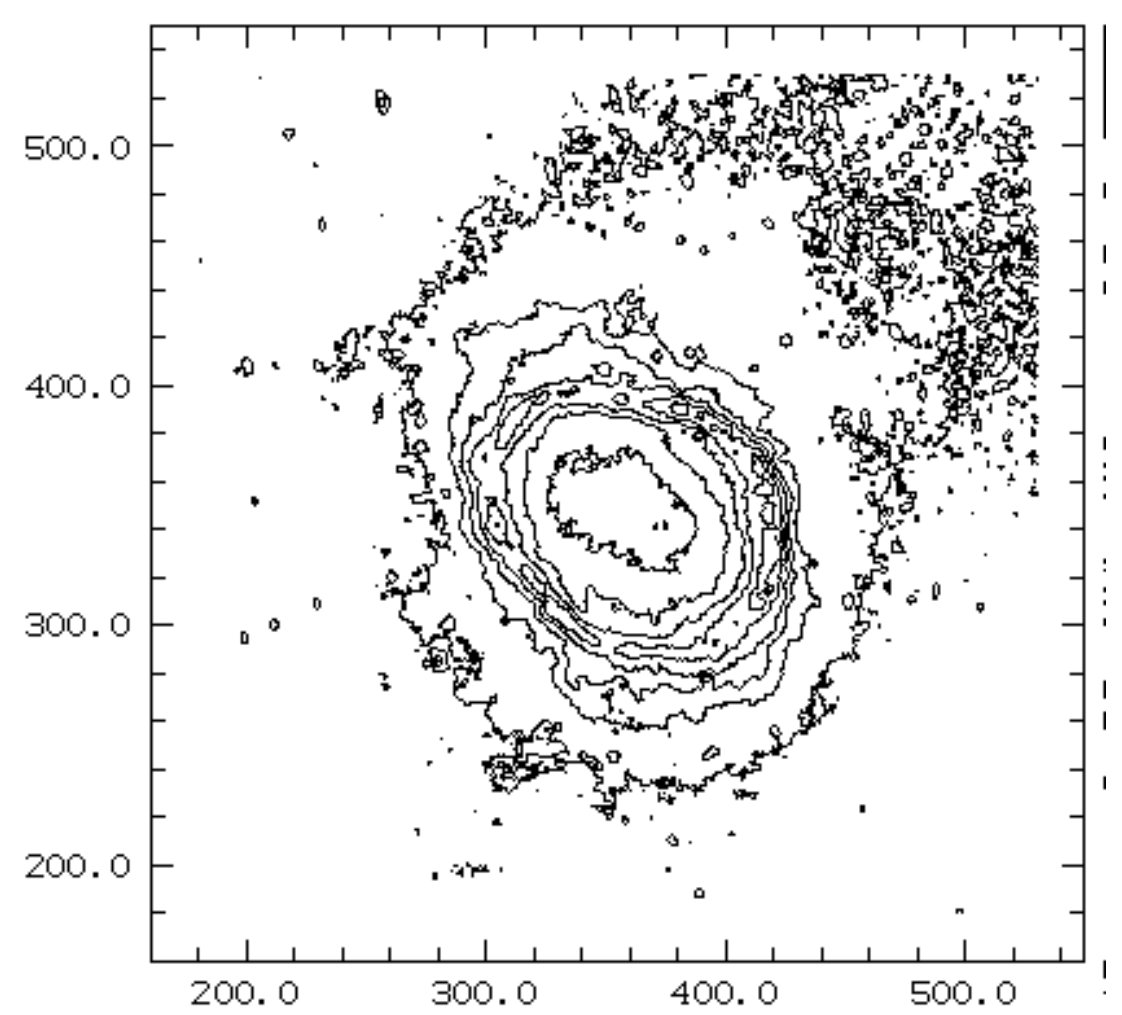

Fig. 5. Isophotal contours of the intensity ratio map with start/end/step $=0.4 / 2.5 / 0.3$, showing that the outer faint extension PA is perpendicular to that of the bright ring (in agreement with Rosado \& Moreno 1991). See A 24 below. Coordinates are in arc seconds. A EW cross-section through the central star (line 340) shows the two peaks separated by $115^{\prime \prime}$

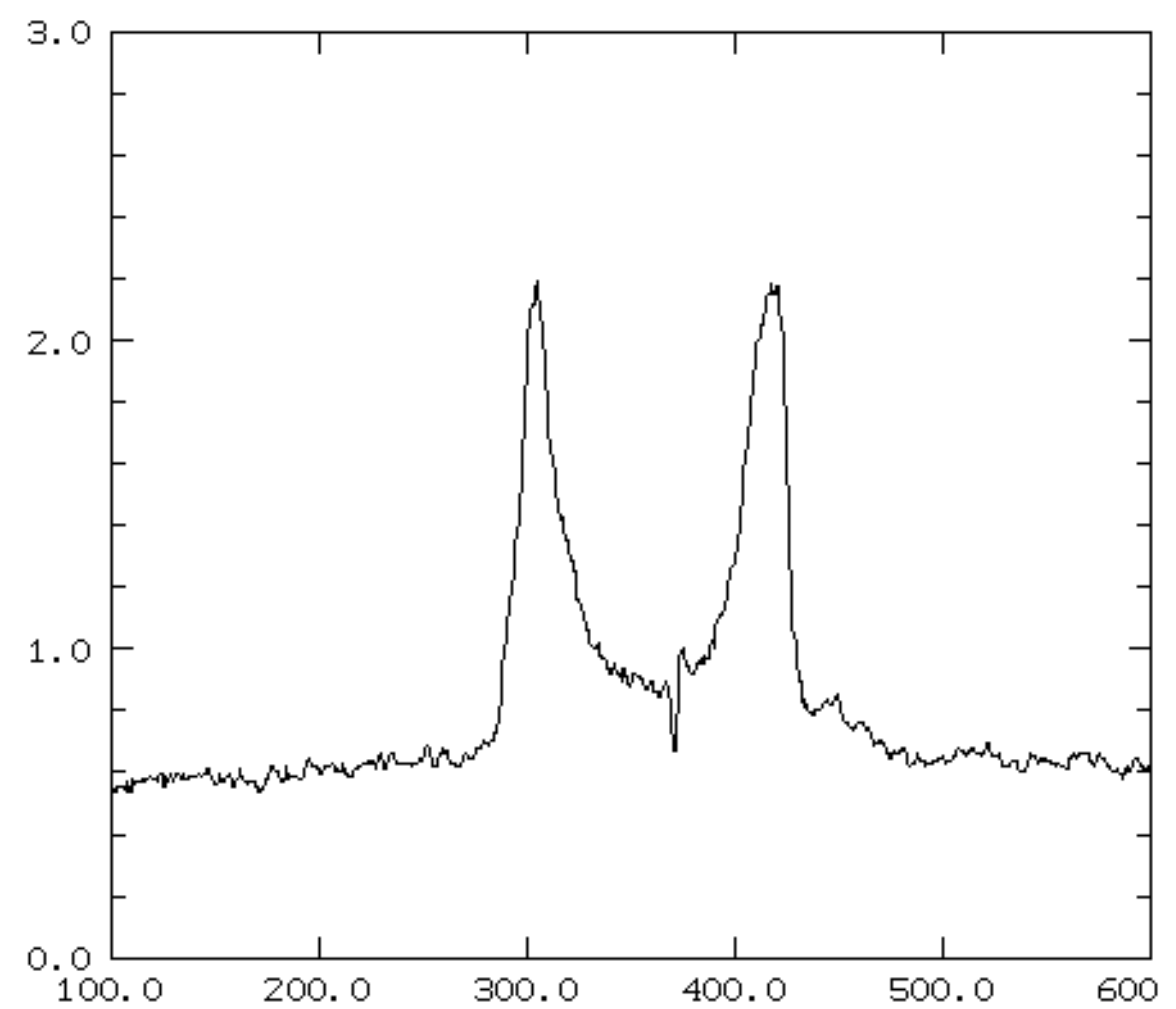

Fig. 6. A EW cross-section through the central star (line 340 ) shows the two intensity ratio peaks separated by $115^{\prime \prime}$ 


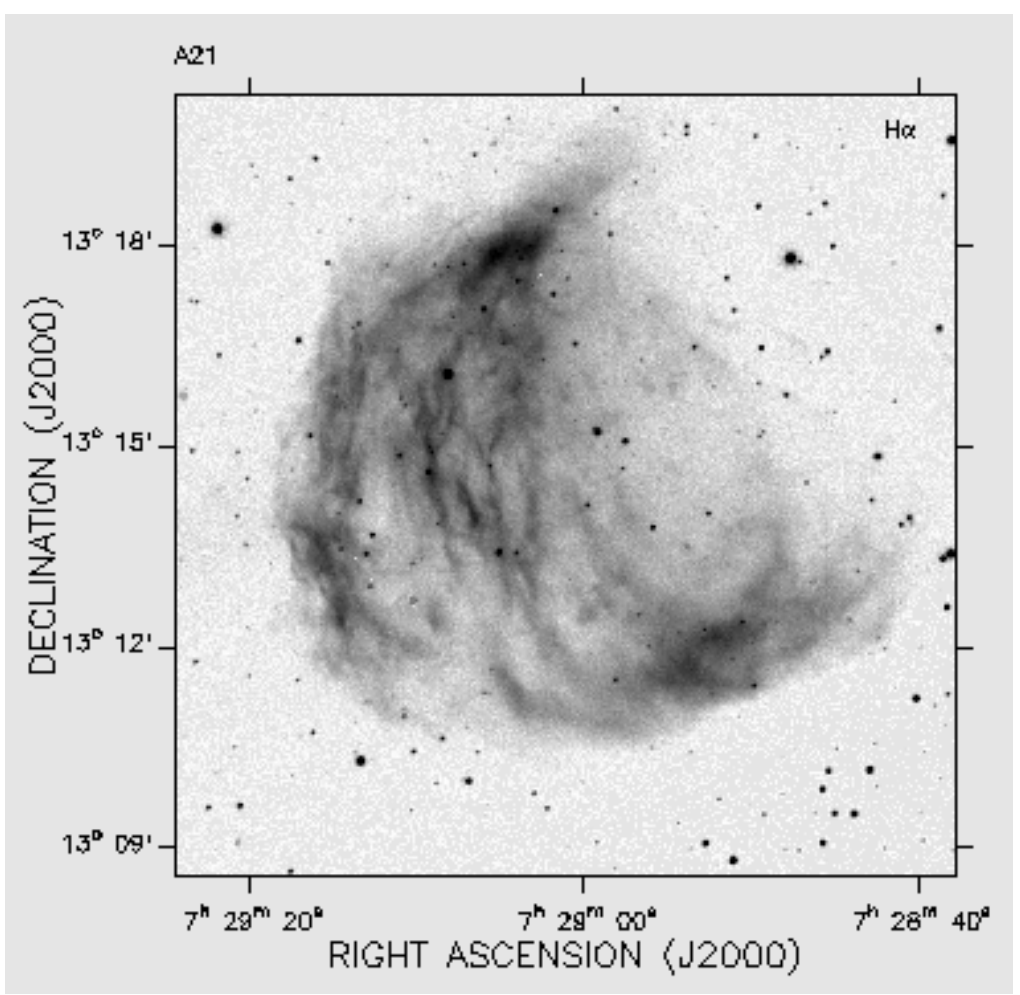

Fig. 7. A 21 in $\mathrm{H} \alpha$ with equatorial coordinates

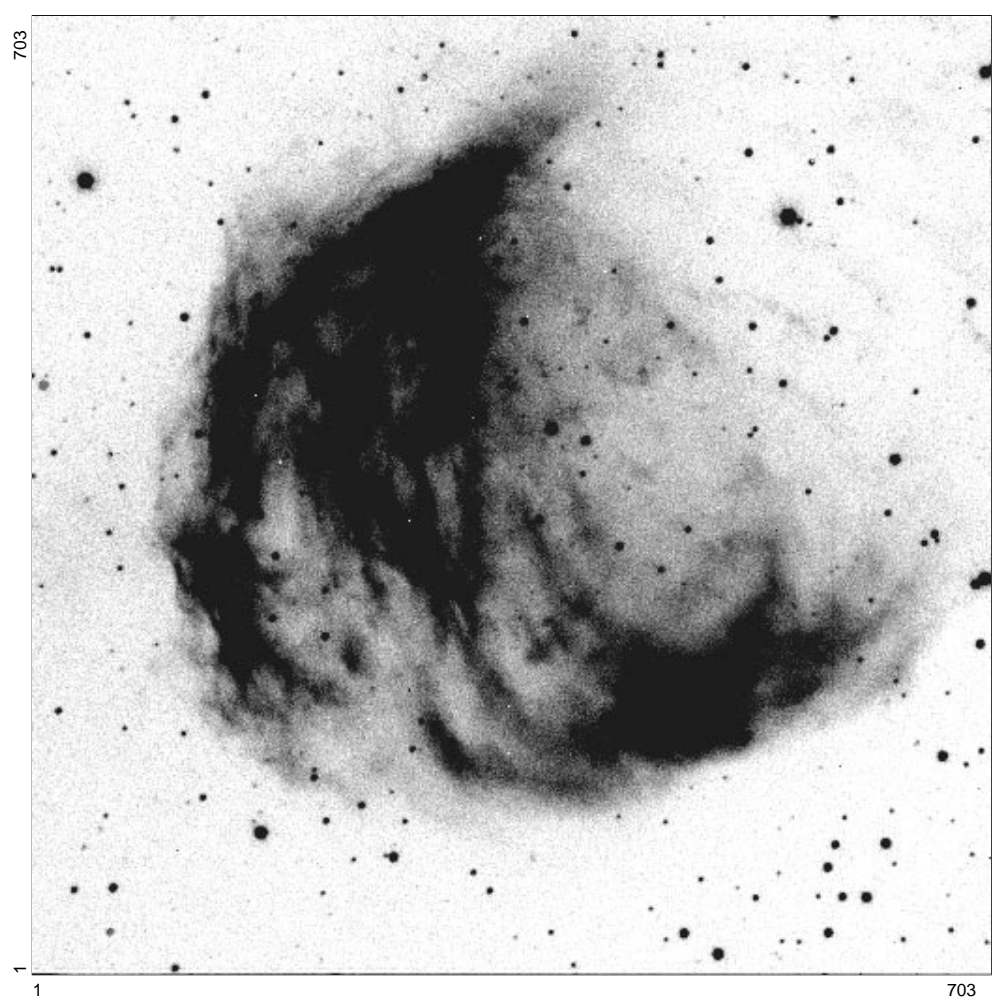

Fig. 8. The overall dimensions of A 21 are certainly much larger than this $\mathrm{H} \alpha$ image spreading over $700^{\prime \prime}$ as indicated in our field of view 


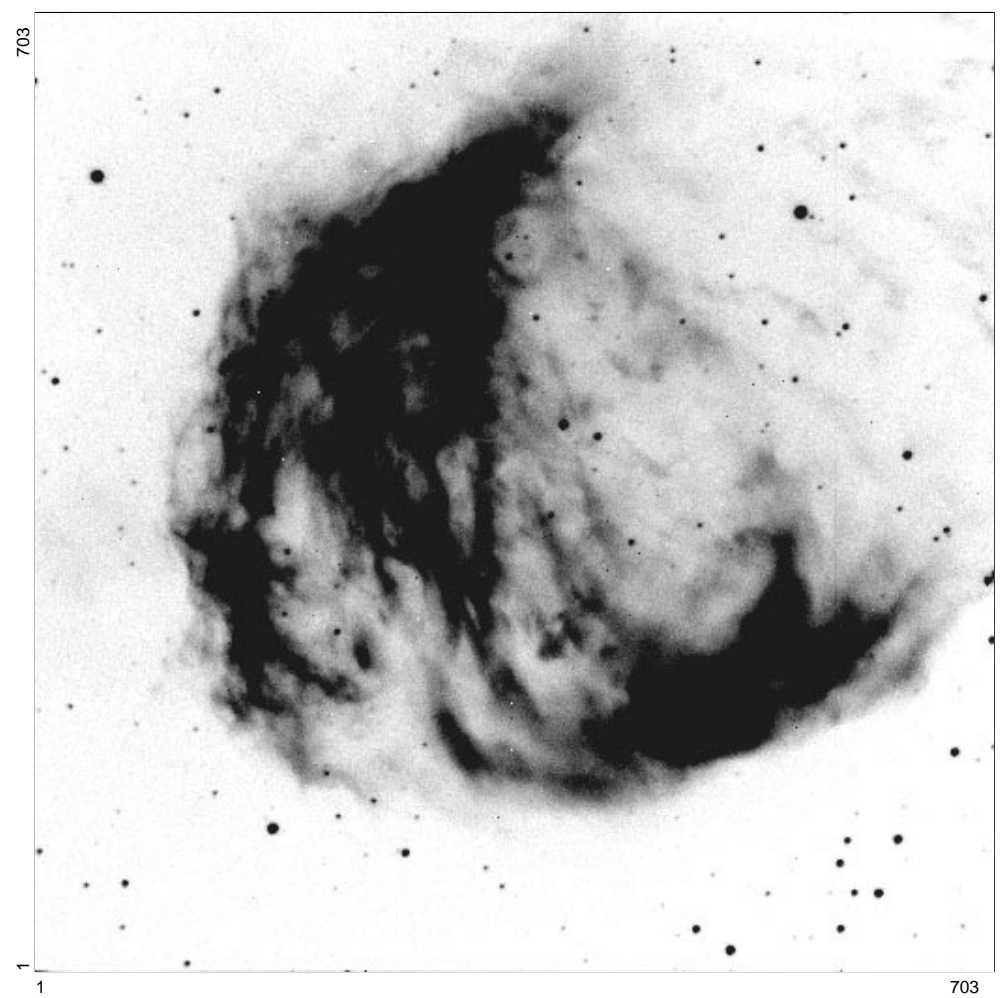

Fig. 9. The [NII] image of A 21 with the same remarks as for $\mathrm{H} \alpha$

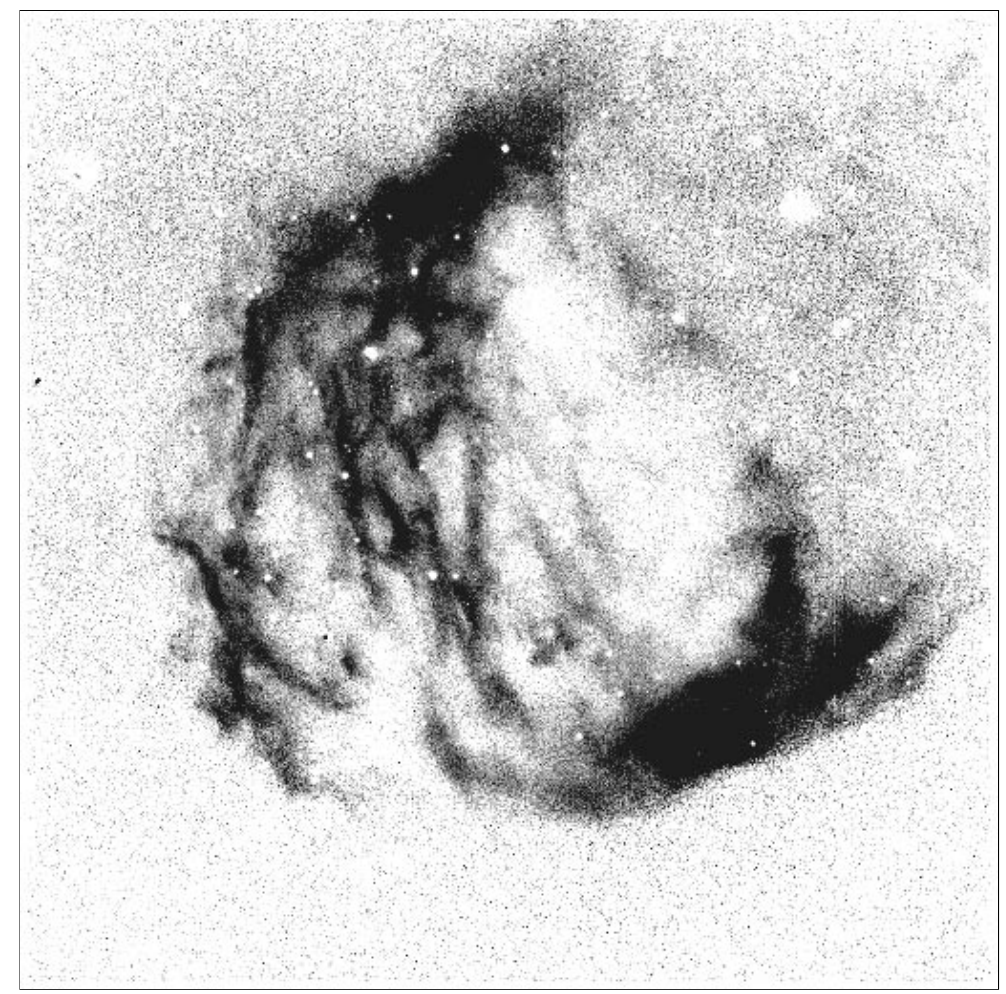

Fig. 10. Variation of the $[\mathrm{NII}]$ to $\mathrm{H} \alpha$ intensity ratio of A 21 


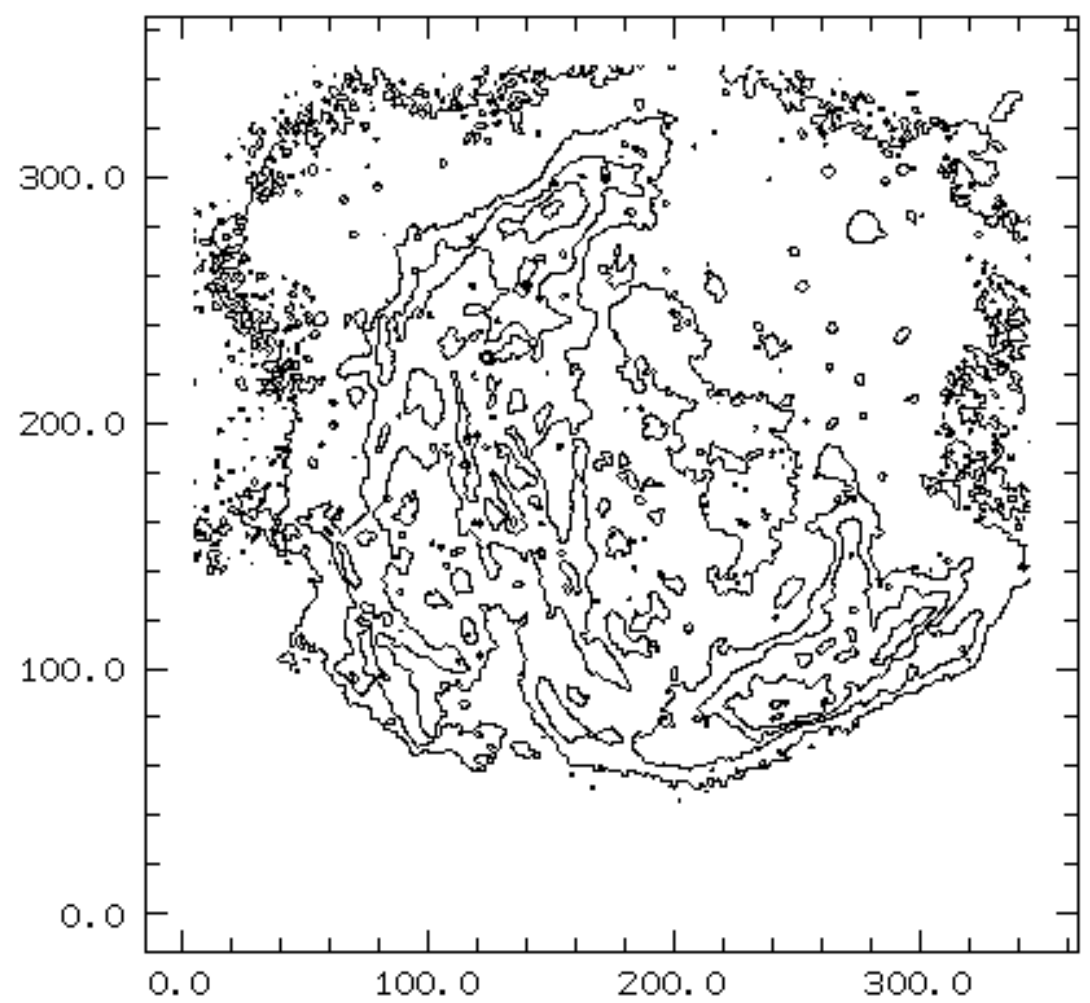

Fig. 11. Isophotal contours for the $[\mathrm{NII}] / \mathrm{H} \alpha$ intensity ratio of A 21 , with 0.7 start, and 0.2 step. Numbers and tick marks are in arc seconds

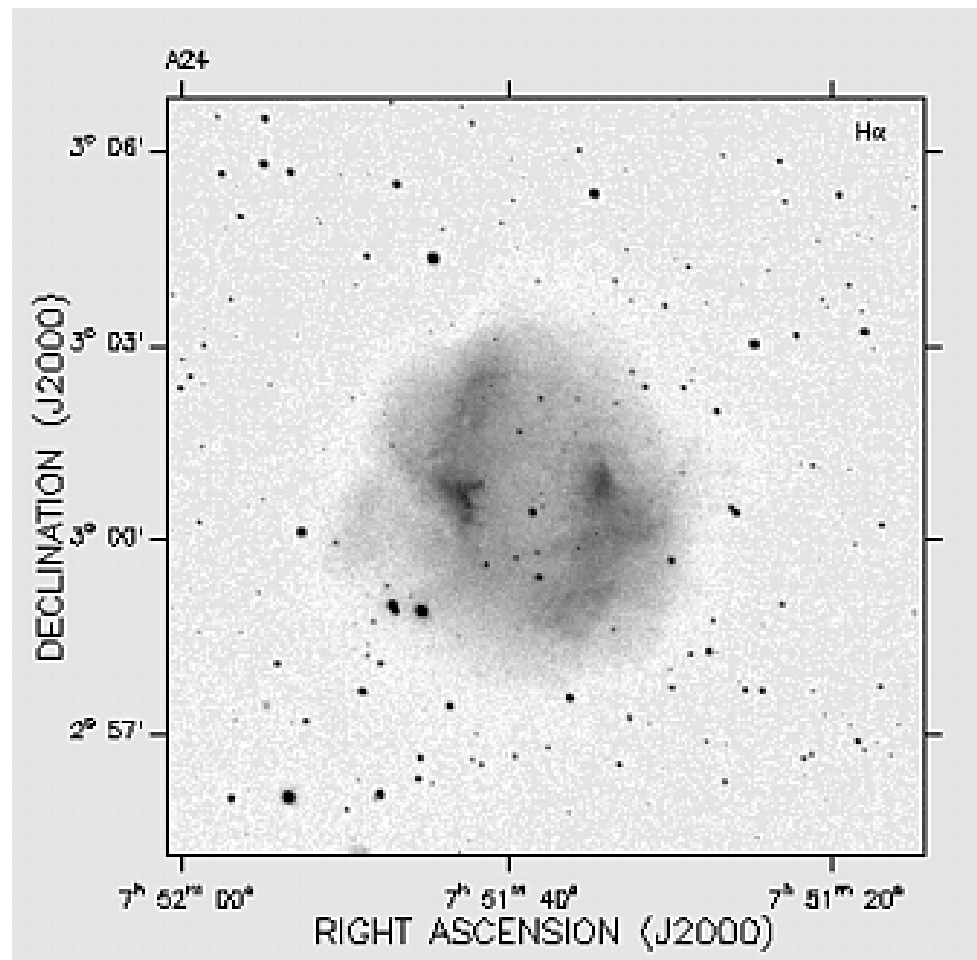

Fig. 12. A 24 in $\mathrm{H} \alpha$ with equatorial coordinates 


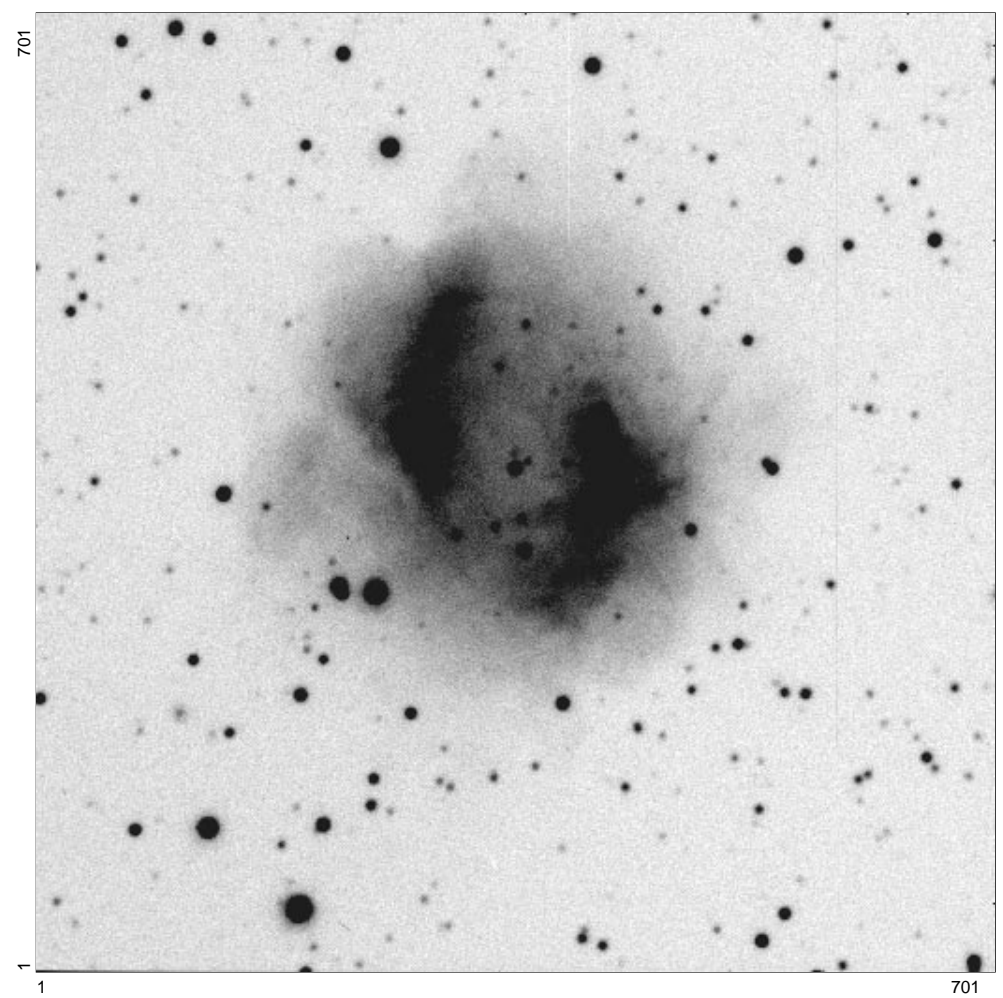

Fig. 13. The $\mathrm{H} \alpha$ image A 24. Numbers are expressed in arc seconds, as for all figures presented

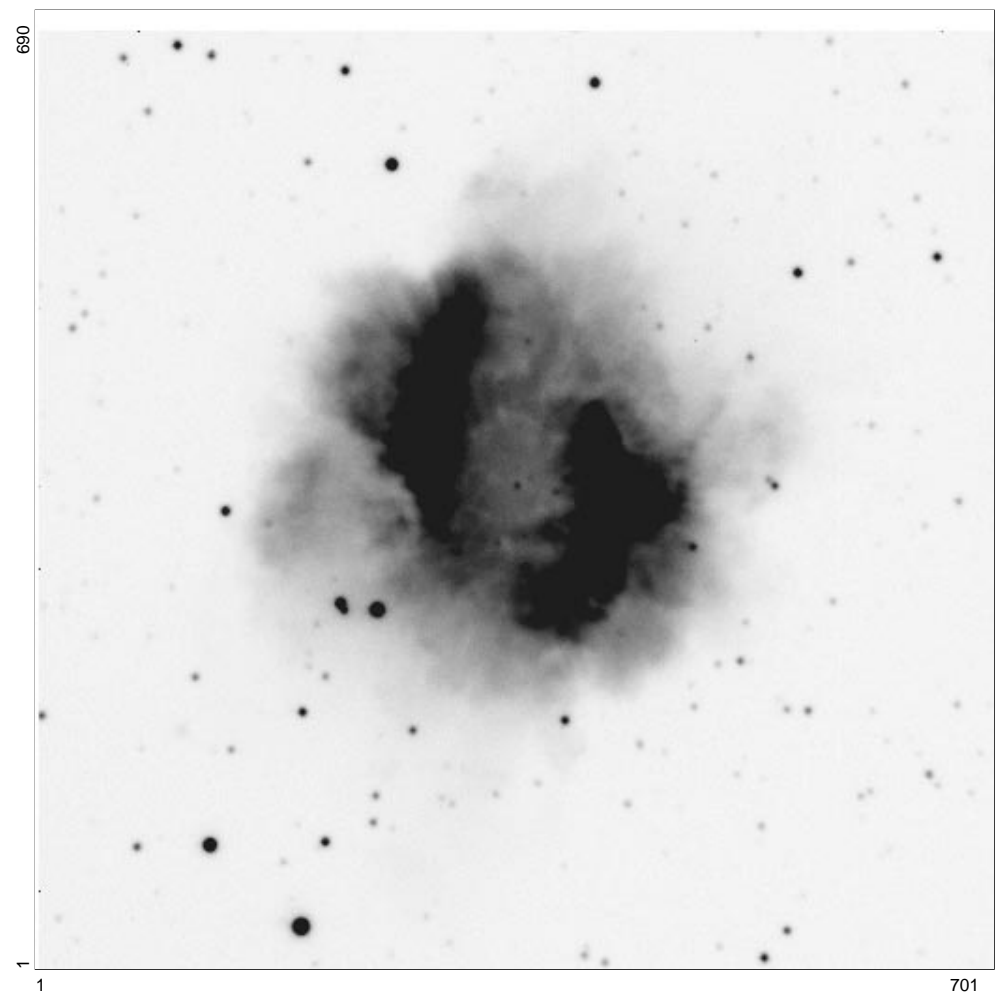

Fig. 14. The $[\mathrm{N} \mathrm{II}]$ image A 24 . Both the lobes and the radial structures are more prominent in the $[\mathrm{N}$ II] than in $\mathrm{H} \alpha$ images 


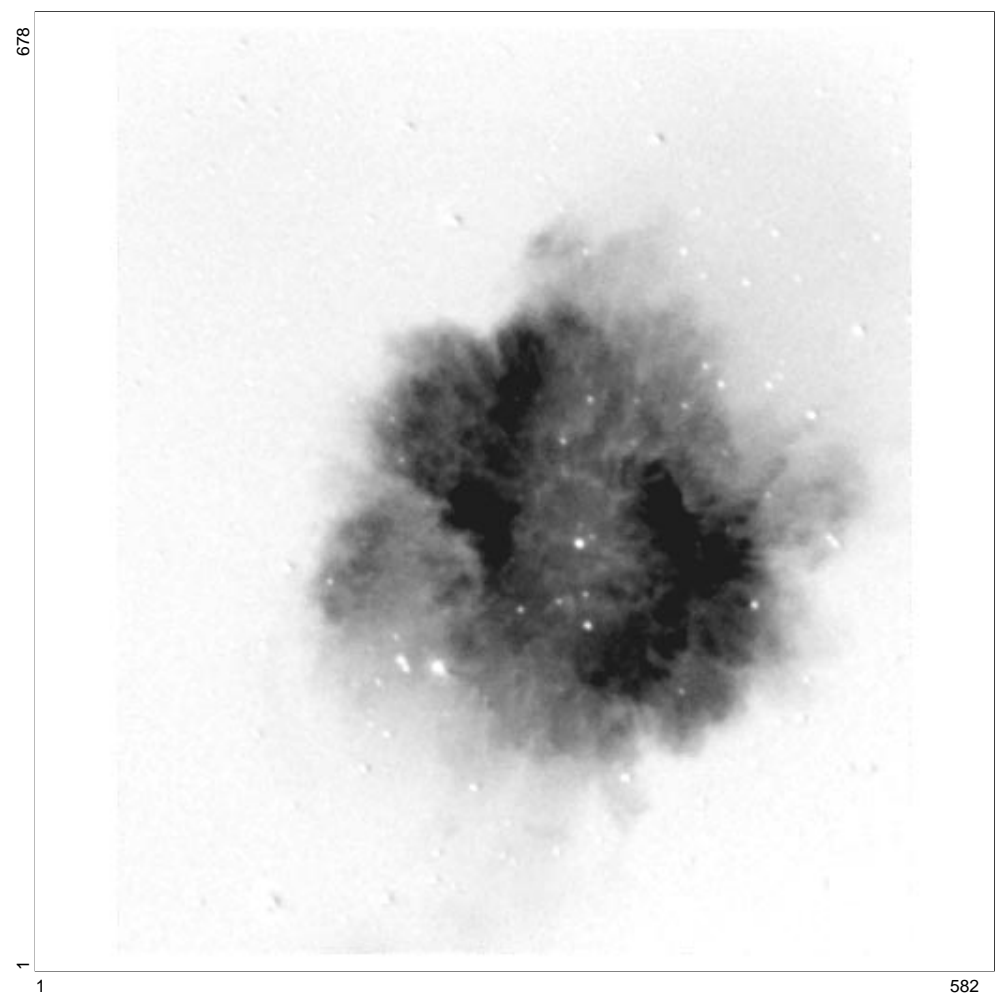

Fig. 15. Ratio of the $[\mathrm{N} \mathrm{II}] / \mathrm{H} \alpha$ images of A 24. This ratio varies from 0.6 in the light shadowed areas to $2.5($ maximum $=3.5)$ within the two lobes

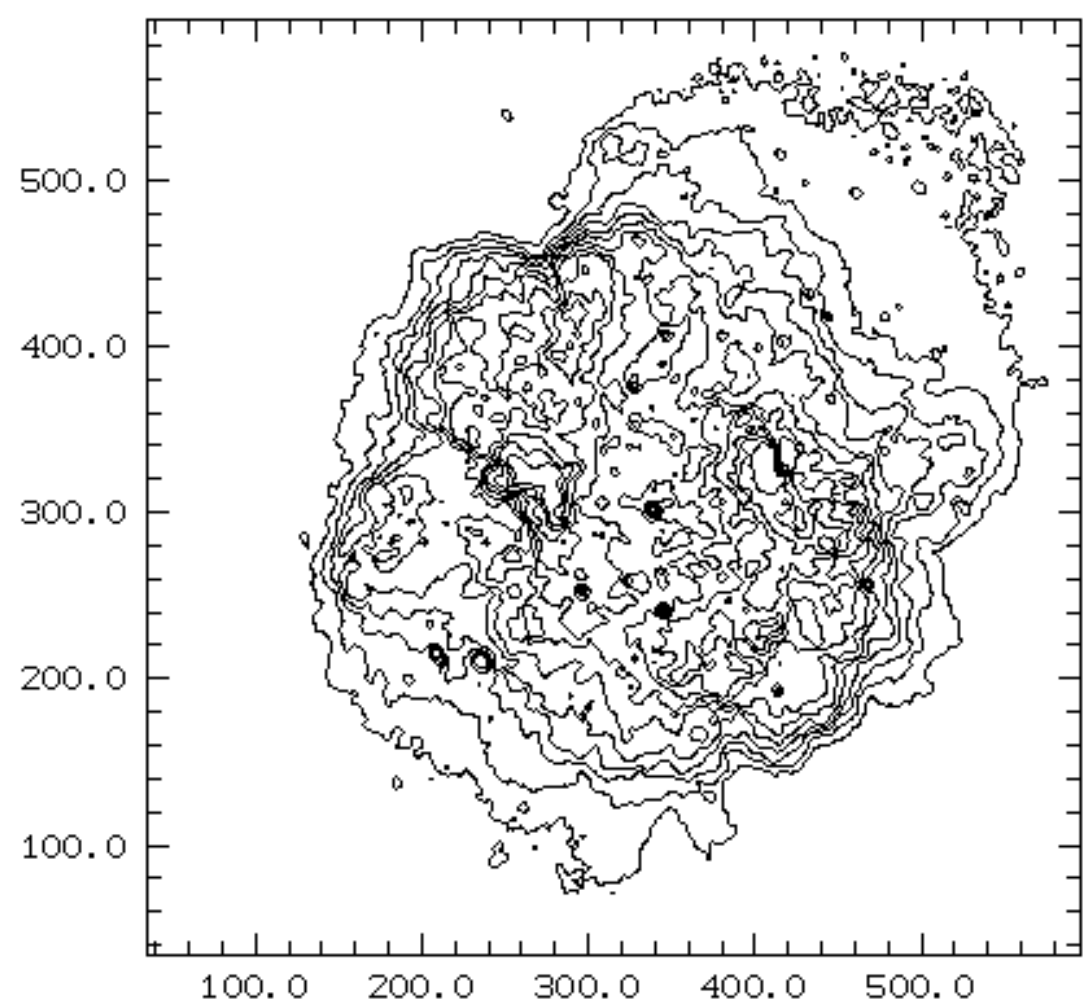

Fig. 16. Isophotal contours (start/step $=0.5 / 0.2$ ) of the $[\mathrm{N} \mathrm{II}] / \mathrm{H} \alpha$ intensity ratio of A 24 . This ratio varies from 0.6 in the light shadowed areas to 2.5 (maximum = 3.5) within the two lobes. A 24 also has the outer extension PA almost perpendicular to that of the bright inner blobs (see above A 13). Coordinates are indicated in arc seconds 


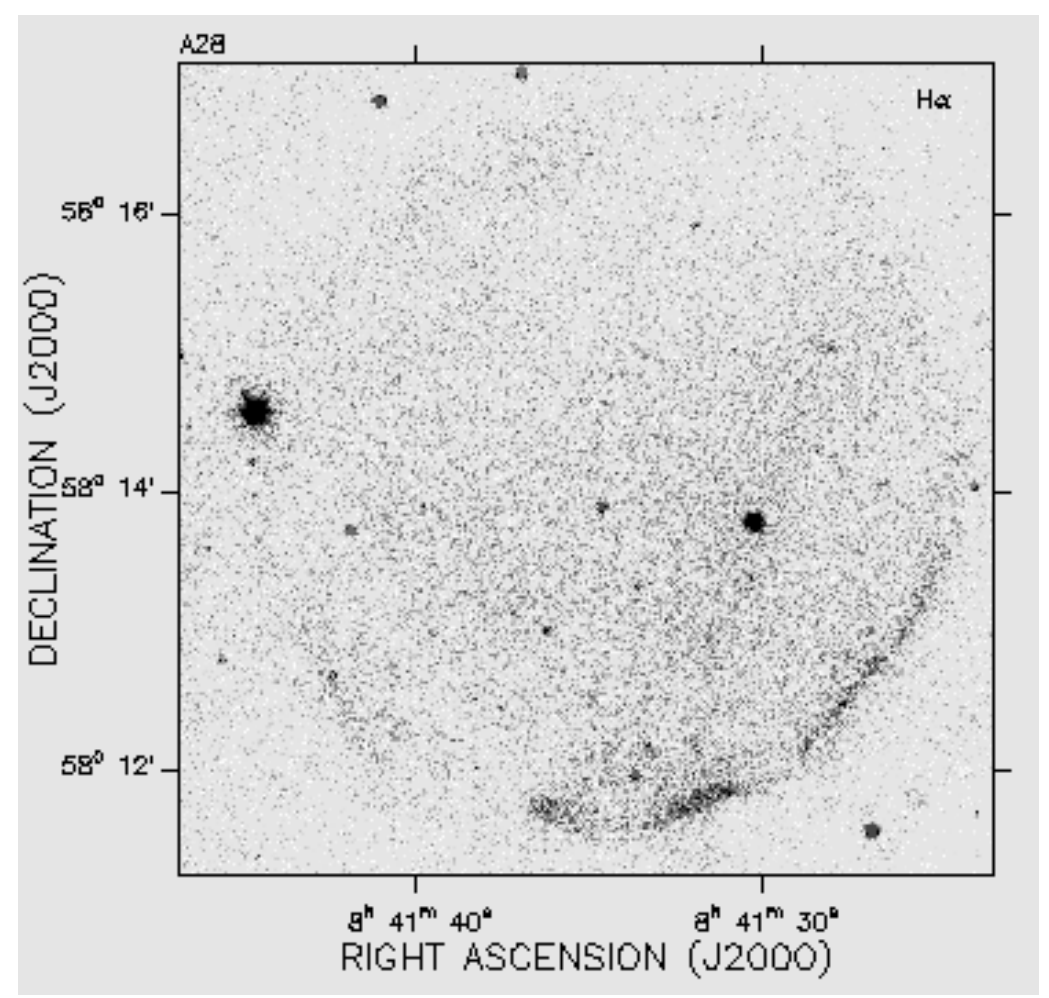

Fig. 17. A 28 with equatorial coordinates

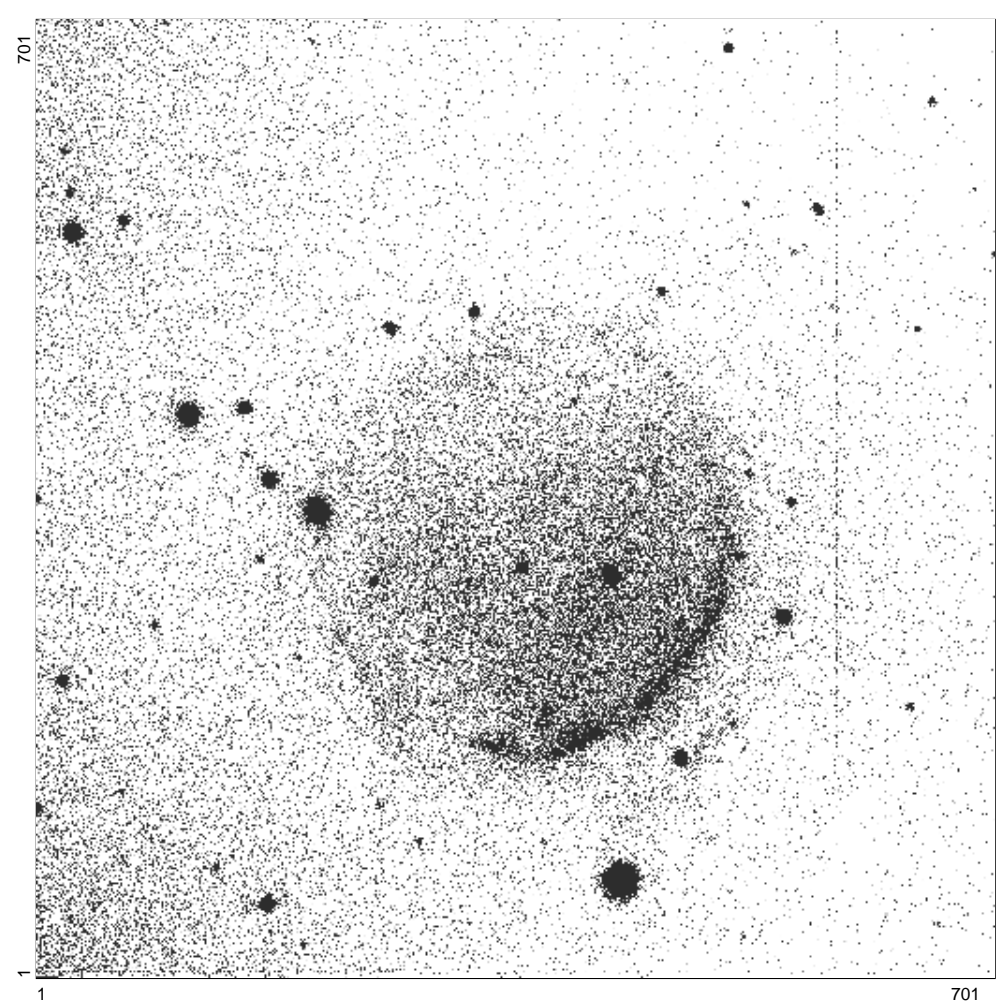

Fig. 18. The $\mathrm{H} \alpha$ image of A 28. Fragments of an outer shell can be seen in the south-west side of this nearly circular PN 


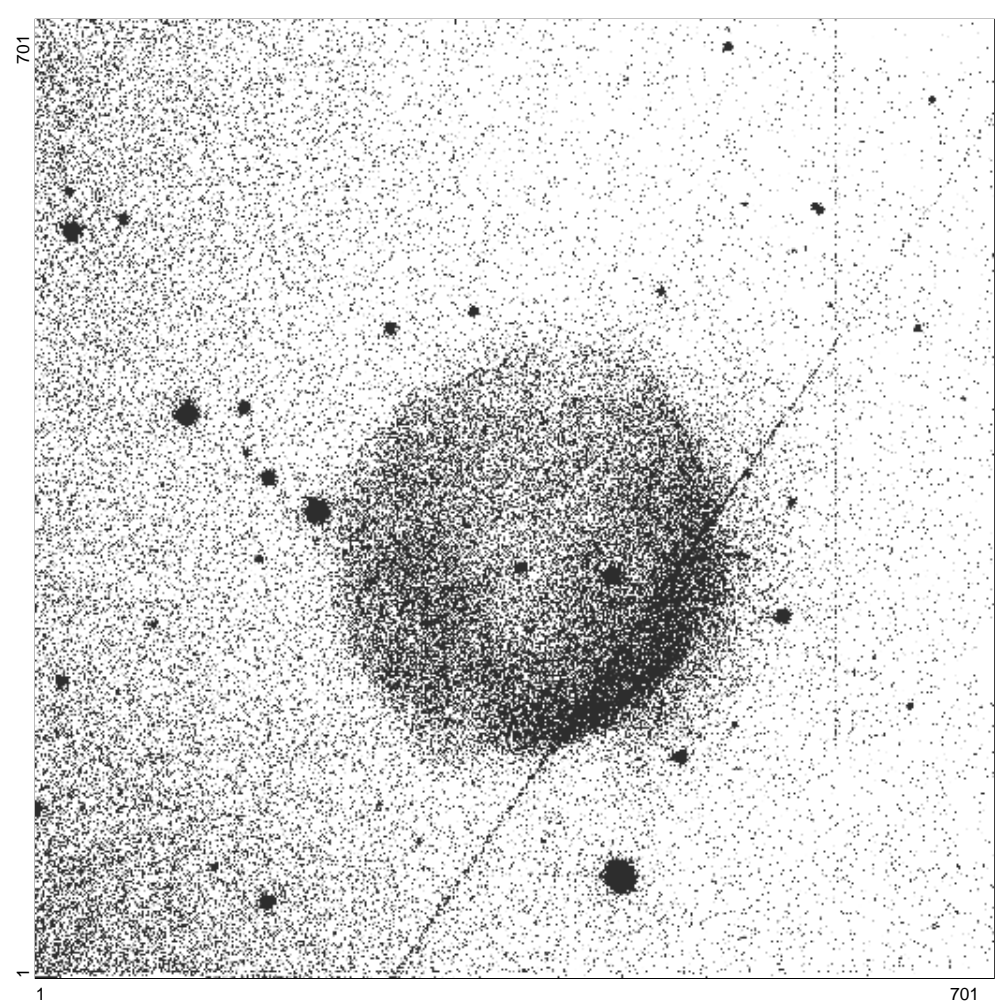

Fig. 19. The [N II] image of A 28 shows a ring-like shape with a bipolar structure. The line from NW to SE is the trace of a satellite trajectory

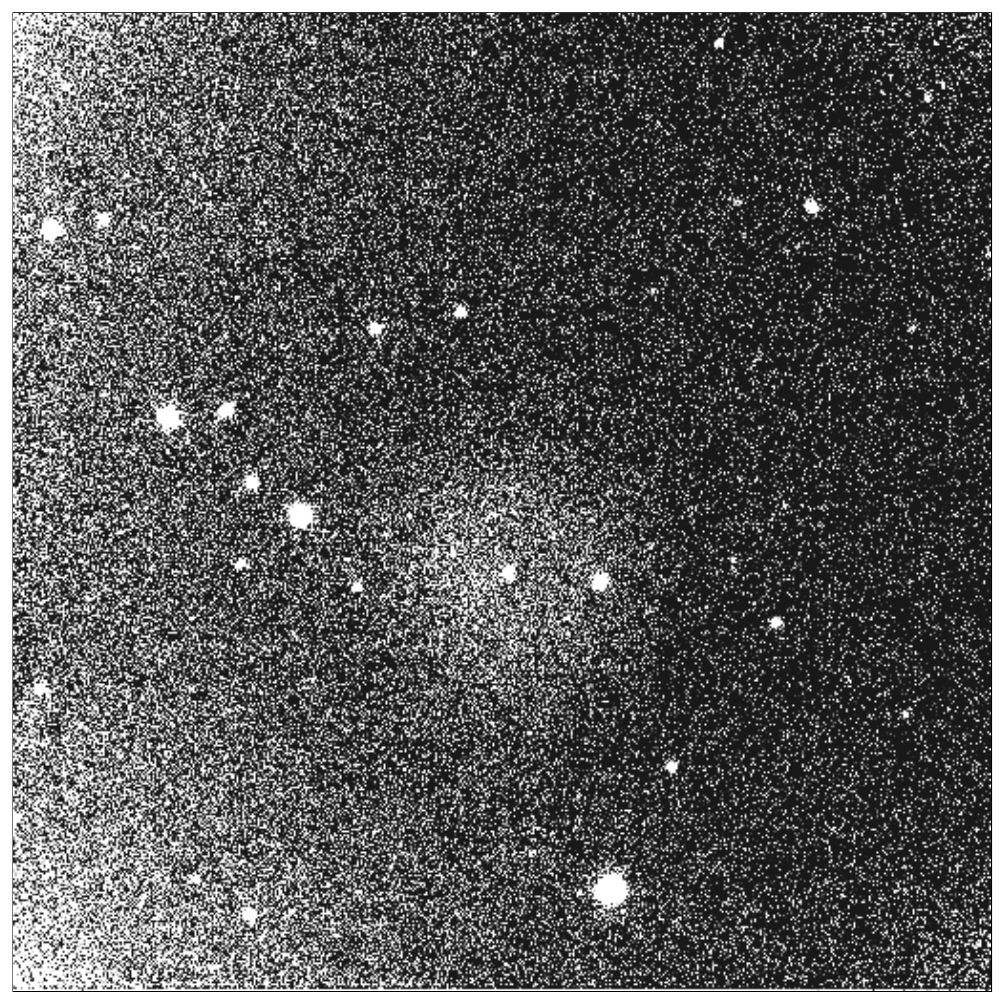

Fig. 20. The $[\mathrm{O}$ III $]$ image of A 28. The $[\mathrm{OIII}]$ emission is faint and well confined to the vicinity of the central star 


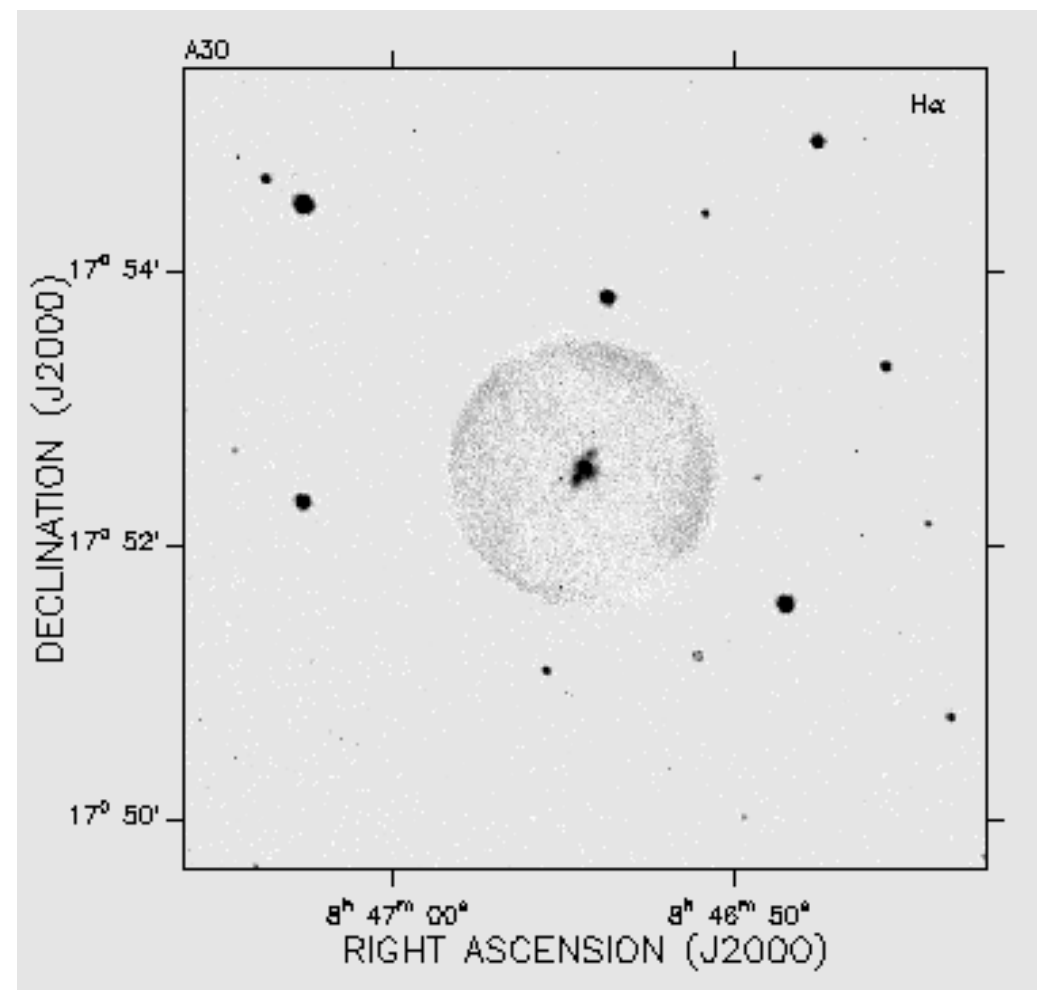

Fig. 21. A 30 with equatorial coordinates

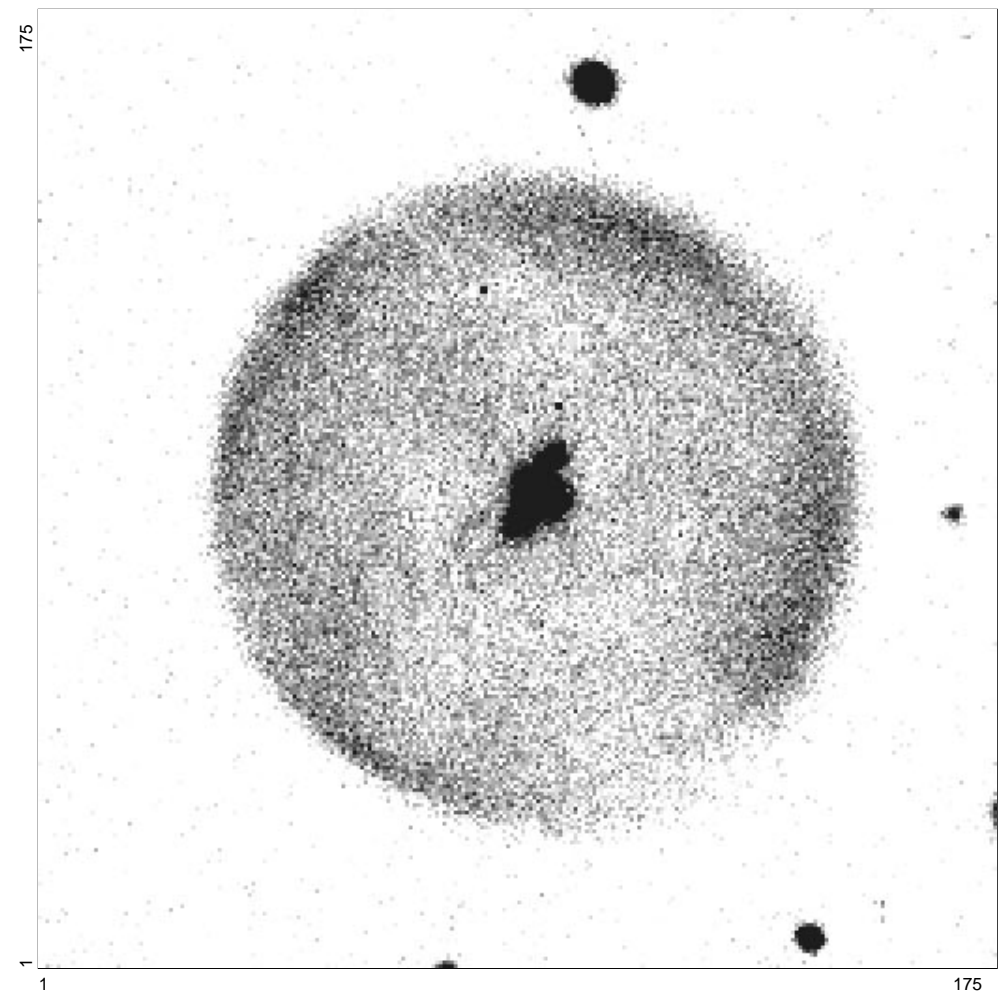

Fig. 22. The $\mathrm{H} \alpha$ image of A 30. The almost perfectly circular shape shows four fragments with enhanced intensity along the ring. The core is composed with two bright knots close to the central star. The [N II] structure only consists of the same "ansae" but much brighter, see isophotes below 


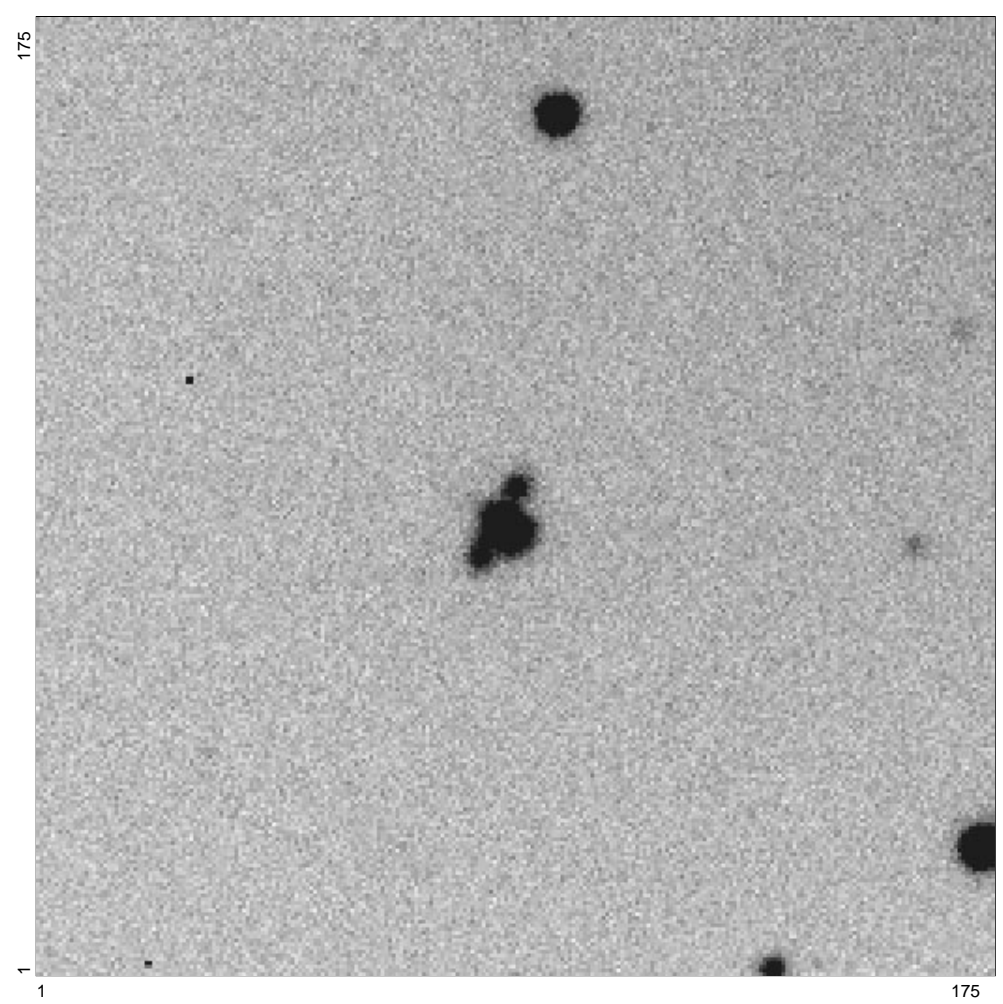

Fig. 23. The [NII] image of A 30: The spherical shell is not detected (even though very faint "traces" can be suspected against the noise level) in the $[\mathrm{N} \mathrm{II}]$ image, suggesting that the shell may be nitrogen poor. The $[\mathrm{N} \mathrm{II}]$ structure only consists of the same "ansae" but much brighter, see isophotes below

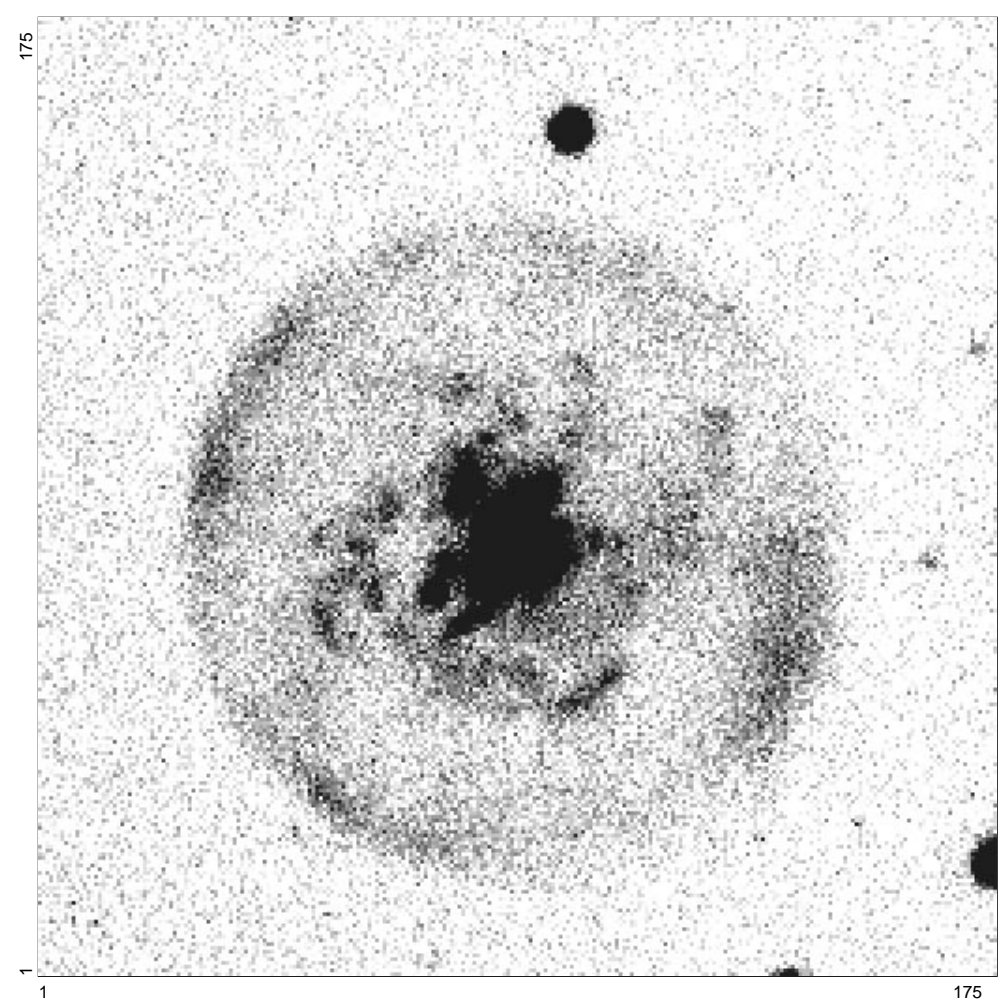

Fig. 24. The $[\mathrm{O}$ III $]$ image of $\mathrm{A}$ 30. The four enhancements along the ring seen in $\mathrm{H} \alpha$ are still visible in [O III]. Yet, the inner "spider" feature seems to whirl around the central knots 

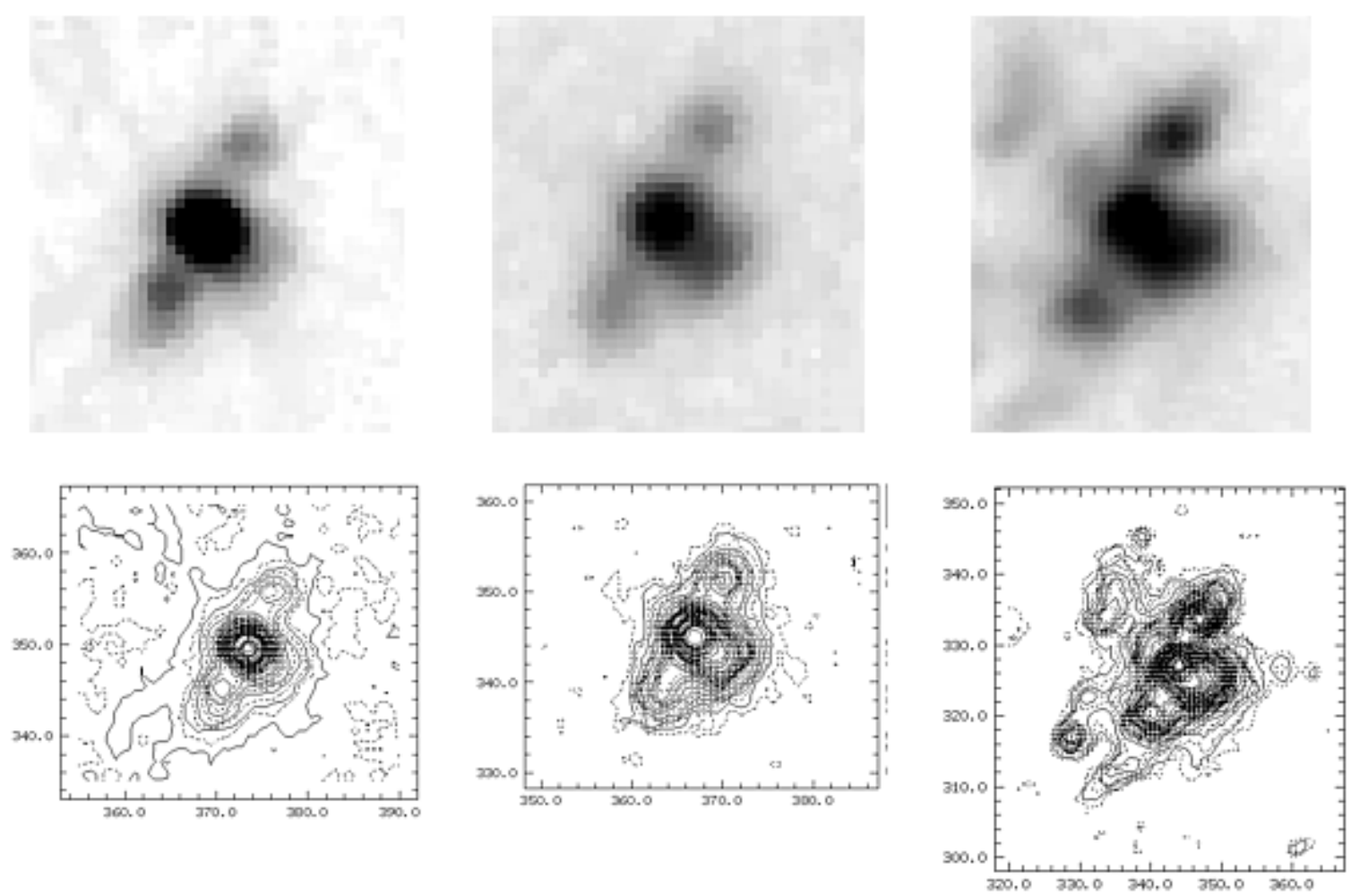

Fig. 25. The central part of A 30 in (from left to right) $\mathrm{H} \alpha$, [N II], and [O III] in grey scale (upper panel) and contour (lower panel) representations. The scales on the contour maps are in arc seconds

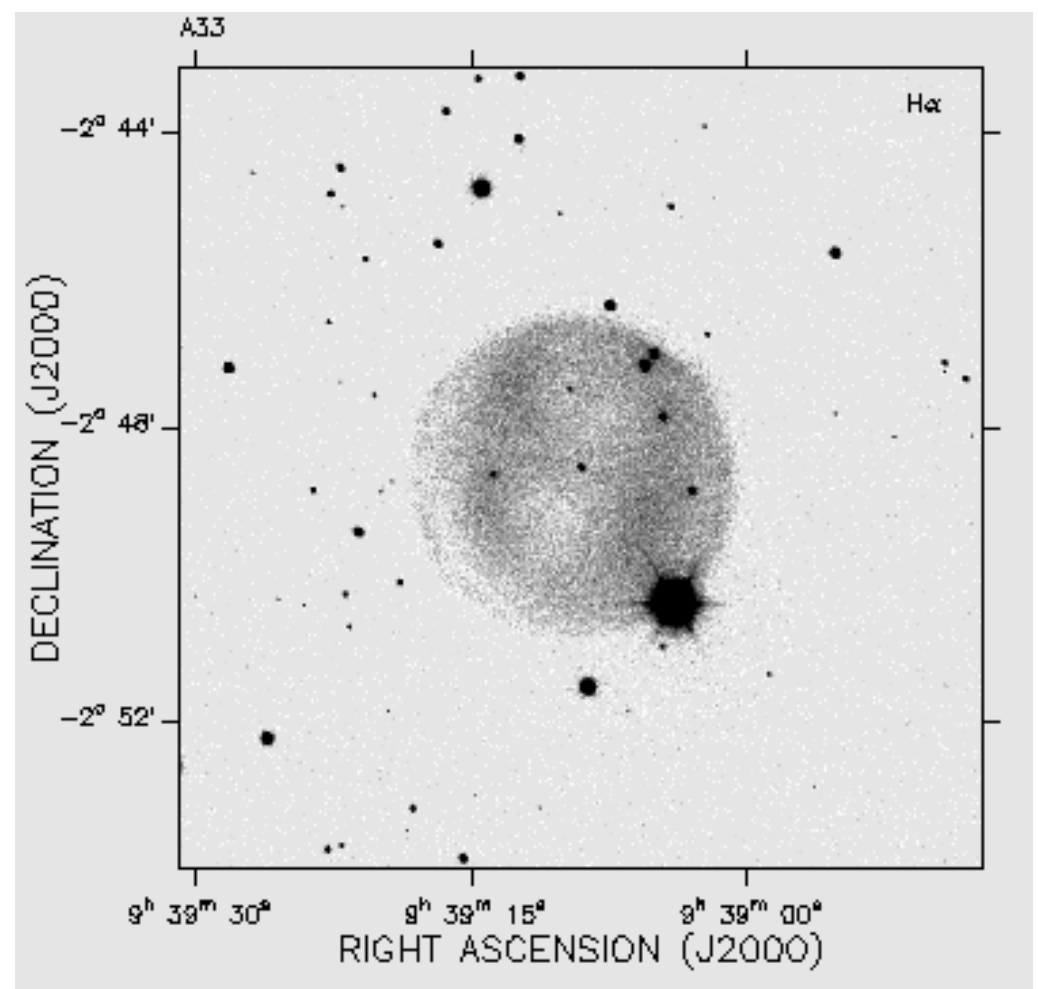

Fig. 26. A 33 with equatorial coordinates 


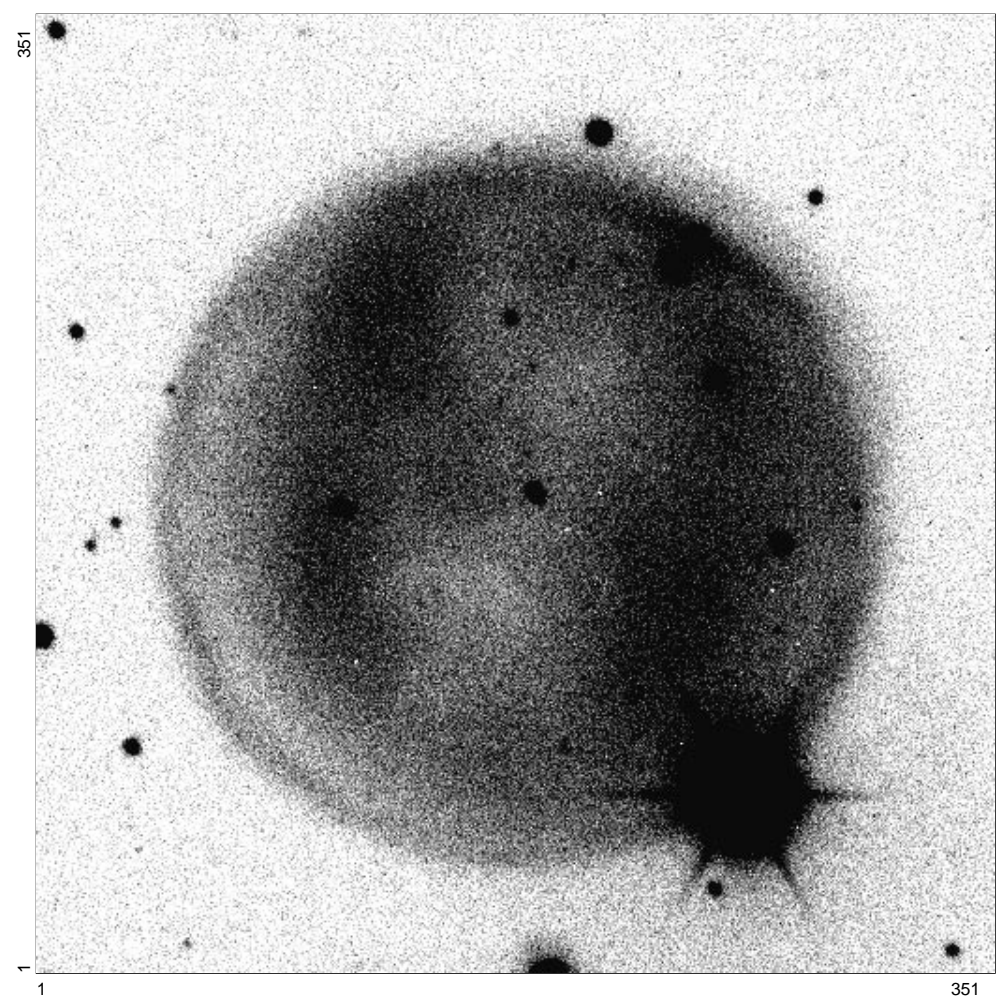

Fig. 27. This $\mathrm{H} \alpha$ image of A 33 nicely illustrates the "diamond ring" structure, while the almost perfectly spherical shape reflects a uniform shell, and the inner structure rather suggests a bipolar morphology

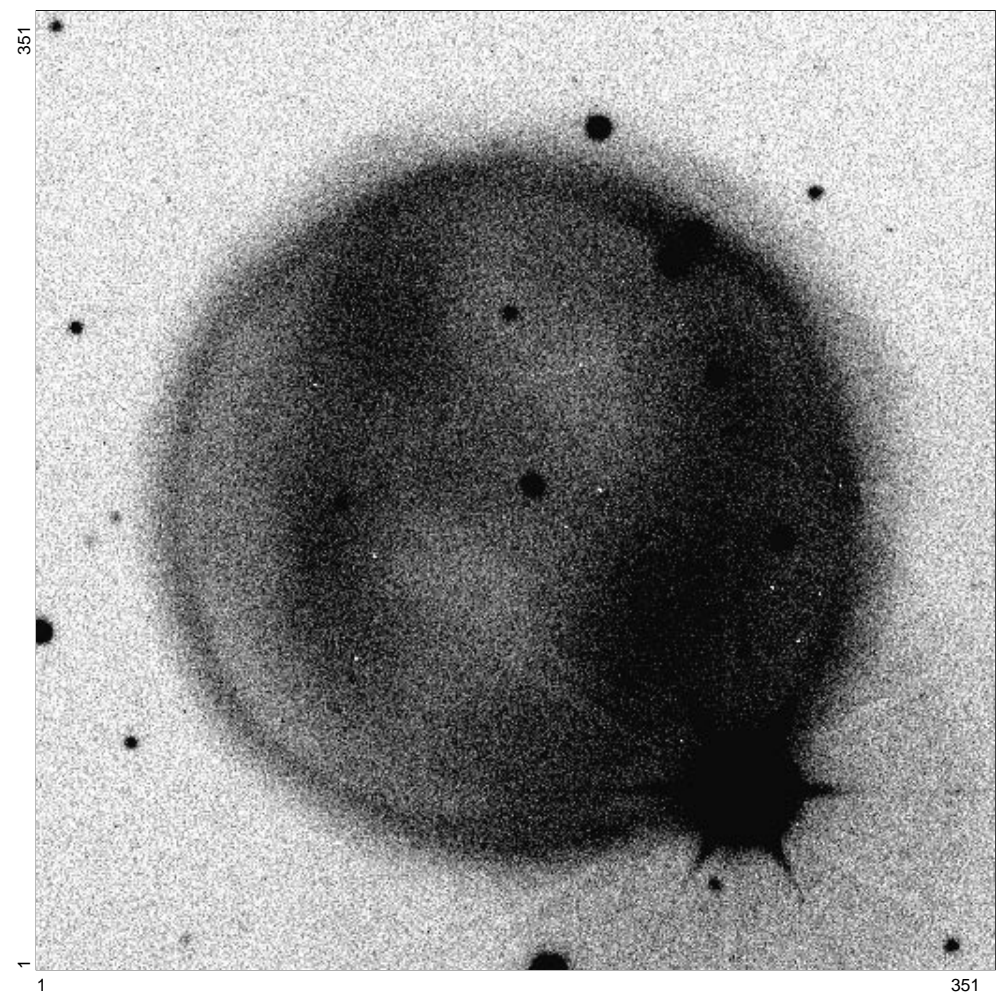

Fig. 28. The [O III] image of A 33 displays a similar structure as for $\mathrm{H} \alpha$, but with a stronger intensity. The inner area resembles NGC 3587, the "Owl Nebula" 


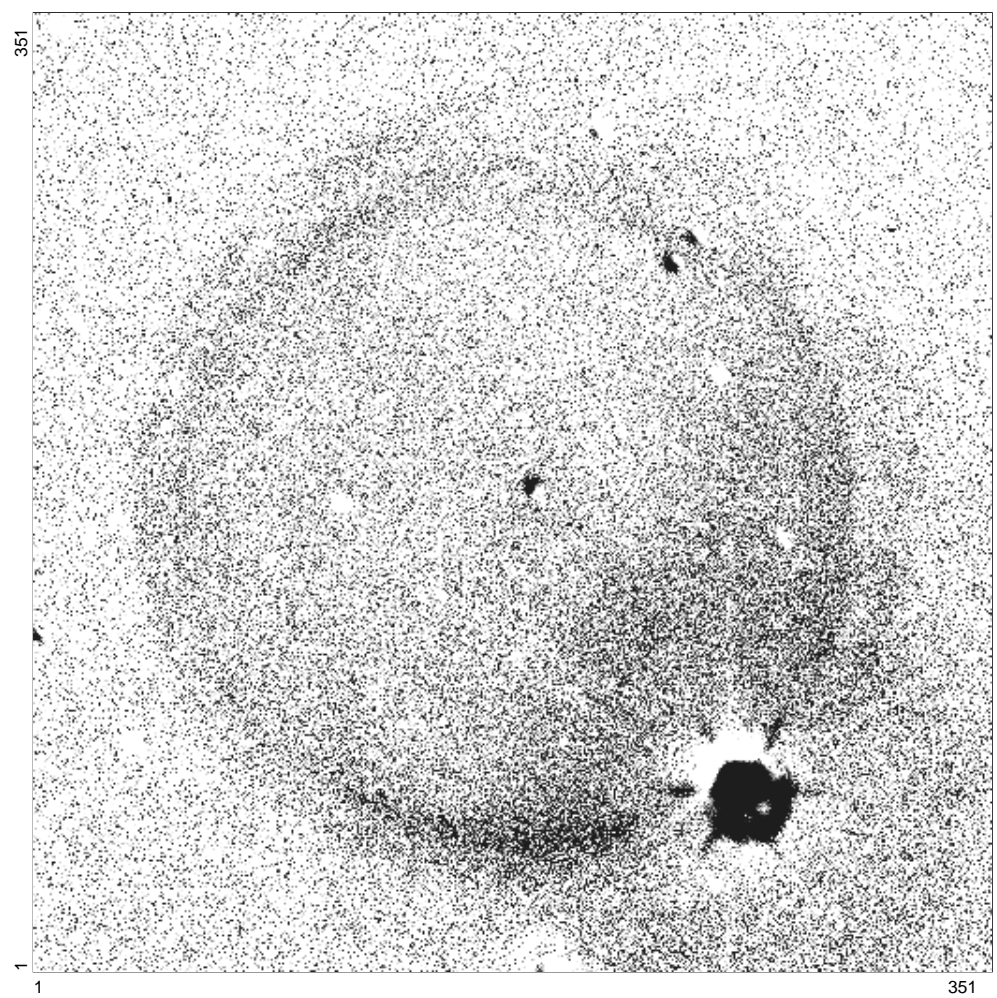

Fig. 29. This $[\mathrm{O}$ III] to $\mathrm{H} \alpha$ intensity ratio of $\mathrm{A} 33$, varying from 0.6 to 1.5 reached on the double-ring structure, reveals a double-shell structure. The circular diffuse emission is due to the bright foreground star

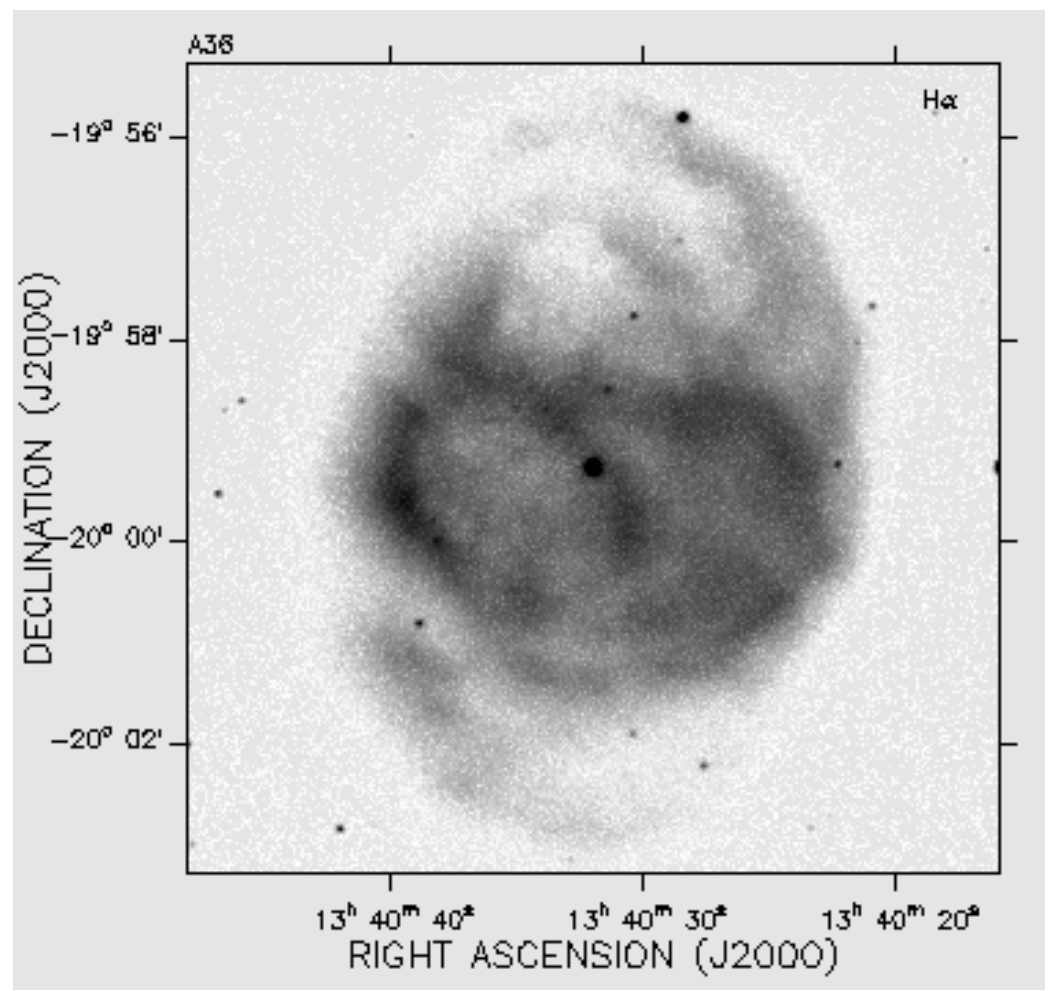

Fig. 30. A 36 with equatorial coordinates 


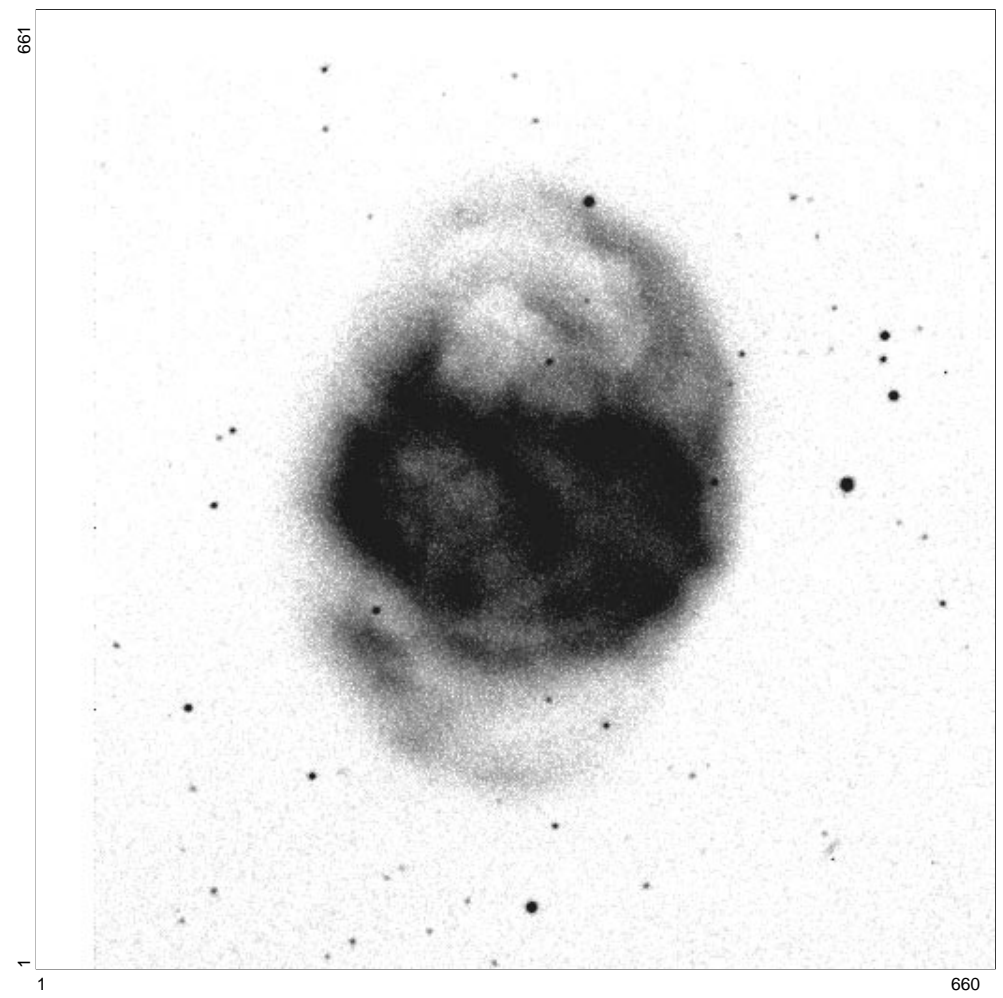

Fig. 31. The $\mathrm{H} \alpha$ image, in logscale, of A 36 shows a helical structure resembling the "Cat Eye" nebula as seen by HST

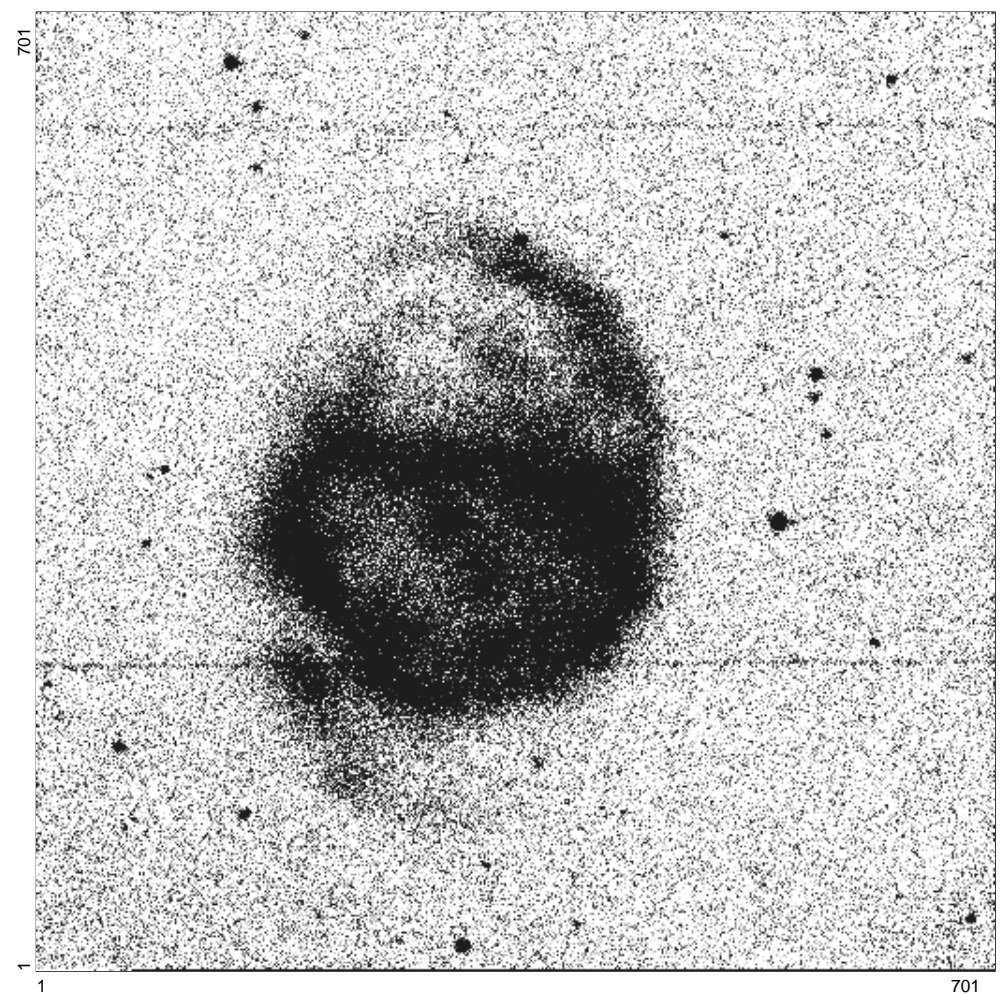

Fig. 32. The $[\mathrm{O}$ III $]$ image of A 36 also displays a similar helical inner region as in the $\mathrm{H} \alpha$ image but with a fainter emission along with outer "spiraling arms" 